Supporting Information

\title{
Resolution of Isomeric Mixtures in Ion Mobility using a Combined Demultiplexing and Peak Deconvolution Technique
}

\author{
Jody C. May ${ }^{1}$, Richard Knochenmuss ${ }^{2}$, John C. Fjeldsted ${ }^{3}$, and John A. McLean ${ }^{1, *}$ \\ ${ }^{1}$ Center for Innovative Technology, Department of Chemistry, Vanderbilt Institute of Chemical Biology, \\ Vanderbilt Institute for Integrative Biosystems Research and Education, Vanderbilt-Ingram Cancer \\ Center, Vanderbilt University, Nashville, Tennessee 37235, United States \\ ${ }^{2} \mathrm{RKR}$ esearch $\mathrm{GmbH}$, Seftigen, Switzerland \\ ${ }^{3}$ Agilent Technologies, Santa Clara, California 95051, United States \\ *To whom correspondence should be addressed. E-mail: john.a.mclean@vanderbilt.edu
}




\section{Contents:}

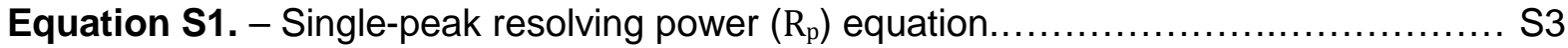

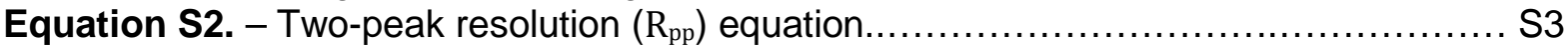

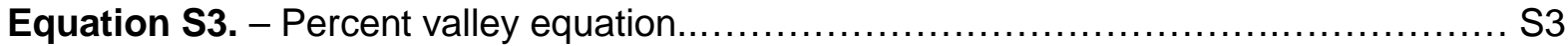

Equation S4. - Peak width at one-tenth the peak maximum.............................. S4

Equation S5. - Percent difference $(\Delta \mathrm{P})$ in drift time between two IM peaks..................S4

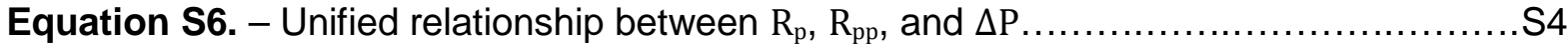

Figure S1. - Simulated IM spectra of two peaks at various resolving powers.............. S3

Figure S2. - Theoretical resolving power curves for the Agilent $6560 \ldots \ldots \ldots \ldots \ldots \ldots \ldots \ldots$ S5

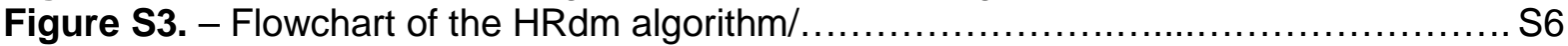

Figure S4. - Information regarding the commercial tuning mixture $\ldots \ldots \ldots \ldots \ldots \ldots \ldots \ldots . . \ldots 7$

Table S1. - Detailed information and sources for all chemical standards................... S8

Table S2. - Drift time, intensity, and signal-to-noise ratios (S/Ns) for tune mix..............S9

Figure S5. - Box-and-whisker plot of the fold change in S/N across drift time...............S13

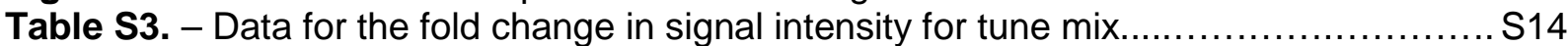

Table S4. - Data for the fold change in the S/N for tune mix ................................. S14

Table S5. - Histogram data for tune mix signal intensity and S/N.......................... S14

Figure S6. - Method for estimating baseline noise in single pulse and 4-bit modes......... S15

Table S6. - Data for baseline noise for single pulse and 4-bit ion multiplexing ............... S16

Figure S7. - Example of improved isotope envelope matching from ion multiplexing ........S17

Table S7. - Corresponding peak height data for the isotope matching example............... S18

Figure S8. - IM overlays of standard and HRdm demultiplexed peaks for tune mix............S19

Figure S9. - 2D IM-MS spectrum of LNFP1/LNFP2 showing spectral artifacts in HRdm.... S20

Table S8. - Tabulated data of all CCS measurements and related statistics..................S21

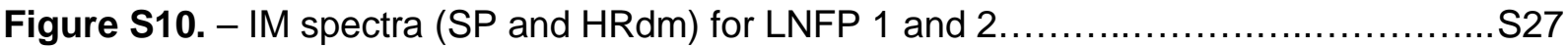

Figure S11. - IM spectra (SP and HRdm) for GD1a and GD1b 34:1 ....................... 28

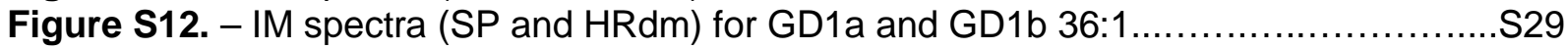

Figure S13. - IM spectra (SP and HRdm) for GD1a and GD1b 38:1 .......................

Figure S14. - IM spectra (SP and HRdm) for 1-AG and 2-AG ........................... 31

Figure S15. - IM spectra (SP and HRdm) for the trisaccharides........................ 32

Table S9 - Drift times, CCS values, $\Delta \mathrm{P}, \mathrm{R}_{\mathrm{p}}, \mathrm{R}_{\mathrm{pp}}$, and \% valleys for all isomer pairs........... S33

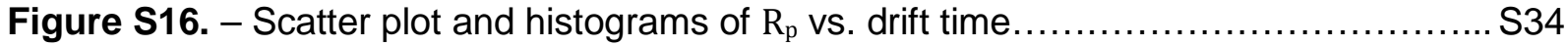

Figure S17. - Histogram of the $\Delta \mathrm{P}$ for a large pool $(\mathrm{N}>3,000)$ of empirical CCS values. ....S35

Appendix A - Protocol for implementing ion multiplexing and HRdm ..................... S36 


\section{Equations used to quantify the observed separation in an ion mobility spectrum: ${ }^{1}$}

The single-peak resolving power $\left(R_{p}\right)$ is calculated from the IM spectrum by dividing the drift time ( $\left.t_{d}\right)$ by the temporal width of the peak $(\Delta t)$, using the full width at half-maximum height (FWHM):

$$
\mathrm{R}_{\mathrm{p}}=\frac{\mathrm{t}_{d}}{\Delta \mathrm{t}}
$$

Equation S1

The two-peak resolution $\left(\mathrm{R}_{\mathrm{pp}}\right)$ is calculated from two IM distributions by dividing the difference in drift times of both peaks by the sum of peak widths and multiplying this quotient by a factor of 1.18 , the latter obtained from the half-maximum definition $(2.355 \sigma / 2)$ :

$$
\mathrm{R}_{\mathrm{pp}}=1.18 \cdot\left|\frac{t_{d 2}-t_{d 1}}{\Delta t_{2}+\Delta t_{1}}\right|
$$

Equation S2

The \% valley separation is estimated from two distinguishable features in the drift time spectrum by dividing the difference between the height of the most abundant feature $(\mathrm{H})$ and the valley height with respect to the baseline $\left(\mathrm{H}_{\mathrm{valley}}\right)$ by the feature height:

$$
\% \text { valley separation }=\left(\frac{\mathrm{H}-\mathrm{H}_{\text {valley }}}{\mathrm{H}}\right) \cdot 100
$$

Equation S3

Thus, when the valley is at the baseline $\left(\mathrm{H}_{\text {valley }}=0\right)$, the valley separation is $100 \%$ (baseline resolved).

The application of these quantitative separation metrics to IM data is shown graphically below for two simulated IM distributions with fixed drift times and varied peak widths:

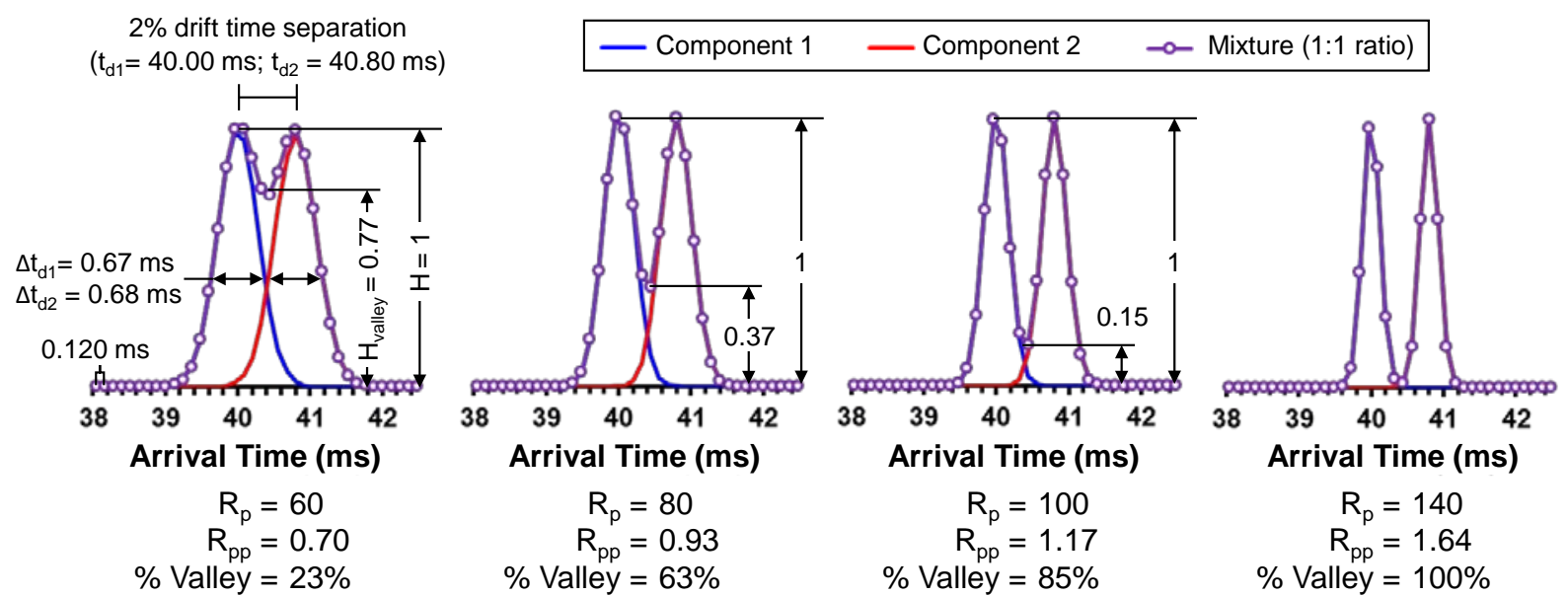

Figure S1. Simulated IM spectra of two normal distributions of equal area and a $2 \%$ separation.

Here, the actual instrument sampling rate of $120 \mu \mathrm{s}$ is simulated, and as peaks narrow from left to right, the simulated spectra indicate that they become less sampled, and this effect is seen experimentally in the HRdm processed results. For context, half-height separation of two peaks occurs at $R_{p p}=0.83$ and baseline separation occurs at $R_{p p}>1.5$. 


\section{Calculation of the number of data points across an IM peak:}

The number of measurements across a peak (Figure 6 of the manuscript) is obtained using the "full width at one-tenth maximum" of the peak in the time dimension $\left(\Delta t_{\text {FWTM }}\right)$, which can be determined from the experimentally-obtained peak width at half height $(\Delta t)$ as follows:

$$
\Delta \mathrm{t}_{\mathrm{FWTM}}=\frac{2 \sqrt{2 \ln 10}}{2 \sqrt{2 \ln 2}} \cdot \Delta \mathrm{t} \approx 1.823 \cdot \Delta \mathrm{t}
$$

This number is then divided by the peak sampling rate $(120 \mu \mathrm{s})$ to determine how many measurements can be made across the peak starting at $1 / 10$ from baseline.

\section{Relating the required ion mobility resolution to the percent difference between two peaks:}

The difference between two peaks $(\Delta \mathrm{P})$ is determined by taking the drift time difference and dividing it by the average drift time, which rearranges as follows:

$$
\Delta \mathrm{P}=\frac{2 \cdot\left|\mathrm{t}_{\mathrm{d} 2}-\mathrm{t}_{\mathrm{d} 1}\right|}{\mathrm{t}_{\mathrm{d} 1}+\mathrm{t}_{\mathrm{d} 2}}
$$

Equation S5

Alternately, the percent difference between two peaks can also be calculated from the known CCS values of analyte ions corresponding to each peak, though some additional error is introduced when comparing two peaks in CCS space as a result of the normalization to CCS via the fundamental low-field ion mobility equation. From the author's experience, this error is small, but introduces $\sim 0.02 \%$ margin of uncertainty ( $\mathrm{N}=44$, Table S9) when comparisons are made across CCS space as opposed to drift time space.

The two-peak resolution $\left(R_{p p}\right)$ can be expressed in terms of peak separation $(\Delta P)$ at half height (i.e., $50 \%$ valley) if the instrument resolving power $\left(R_{p}\right)$ is known by combining Eqns $S 1, S 2$ and $S 5:^{2}$

$$
\mathrm{R}_{\mathrm{pp}}=\left(\frac{\sqrt{2 \cdot \ln 2}}{2}\right) \cdot \mathrm{R}_{\mathrm{p}} \cdot \Delta \mathrm{P}
$$


(A) DTIMS Resolving Power Curves

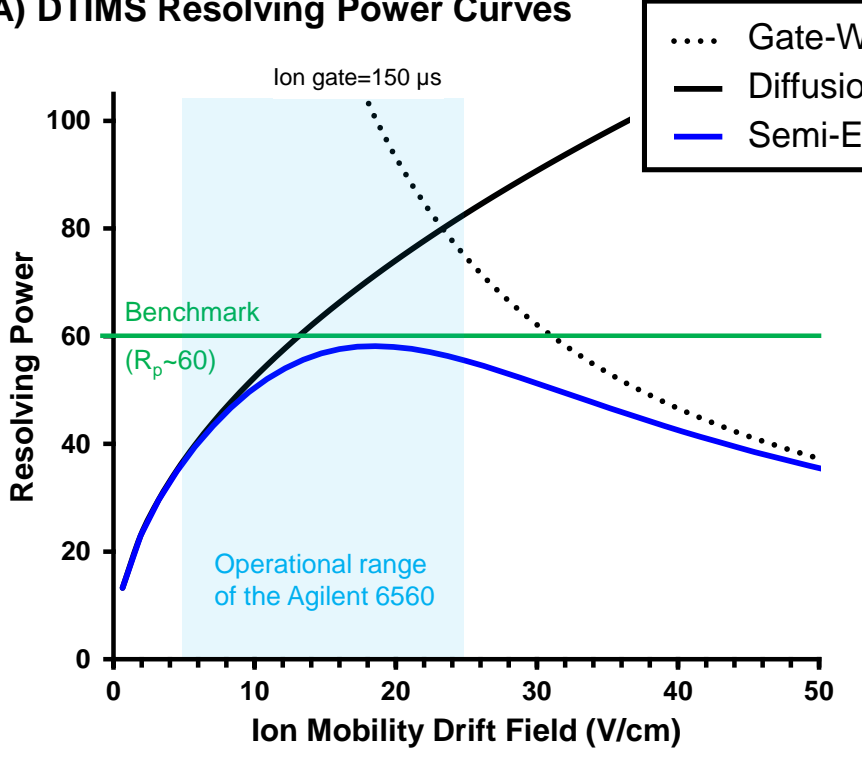

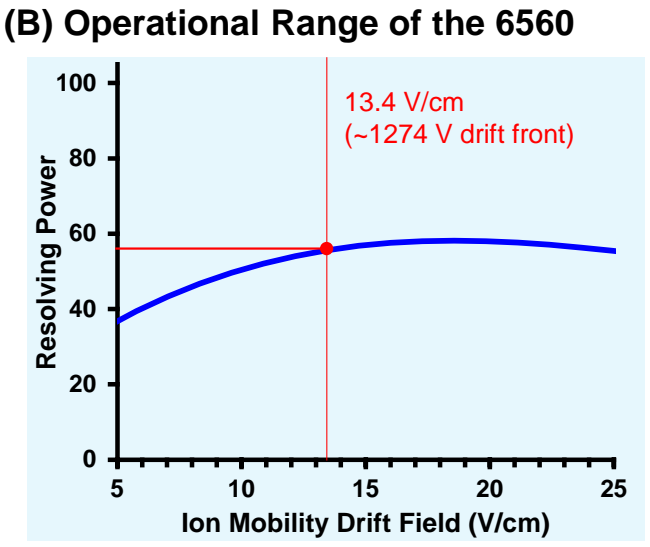

Figure S2. (A) Theoretical resolving power curves for singly-charged ions in drift tube ion mobility instrumentation, ${ }^{3}$ and (B) the operational range of the Agilent $6560 .{ }^{4}$ The blue trace corresponds to the semi-empirical model which is trained against experimental data and indicates the maximum instrument resolving power ( $\sim 60)$ is accessed in the drift field range of ca. 12 to $25 \mathrm{~V} / \mathrm{cm}$. The red lines indicate the drift field used in this study $(13.4 \mathrm{~V} / \mathrm{cm})$ which represents low drift field conditions (extended ion drift times) in which high $R_{p}$ values can still be achieved. 


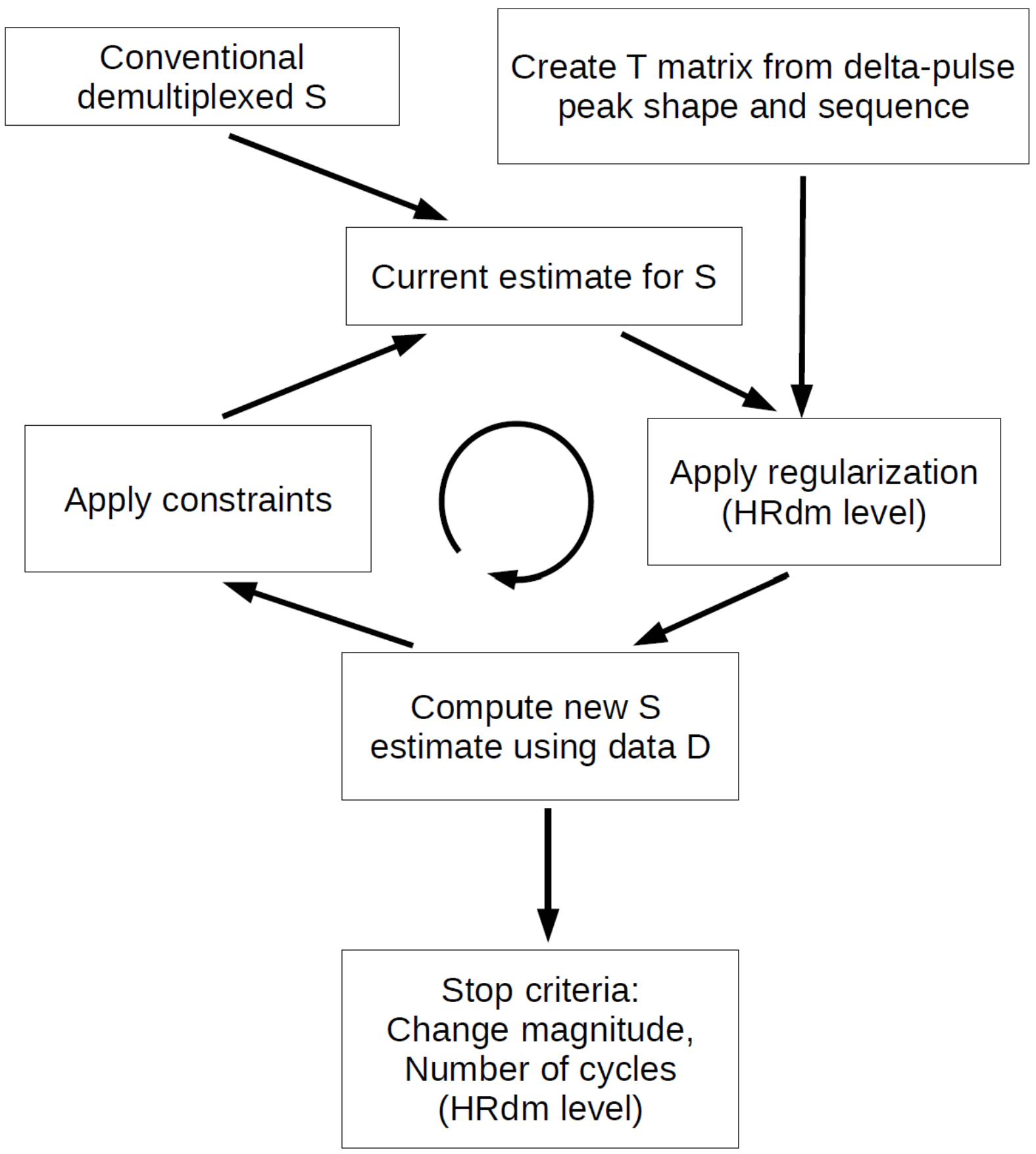

Figure S3. Flowchart of the HRdm algorithm. The starting point is the conventional low resolution demultiplexed spectrum. Subsequent refinements exhibit higher resolution, but the process cannot be continued indefinitely since noise and artifacts eventually become unacceptable. This degradation is also slowed by application of regularization and consistency constraints. Stopping and regularization parameters can be chosen to favor resolution or reduced noise: These are predefined as HRdm "levels". 
betaine

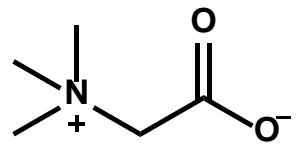

phosphazenes

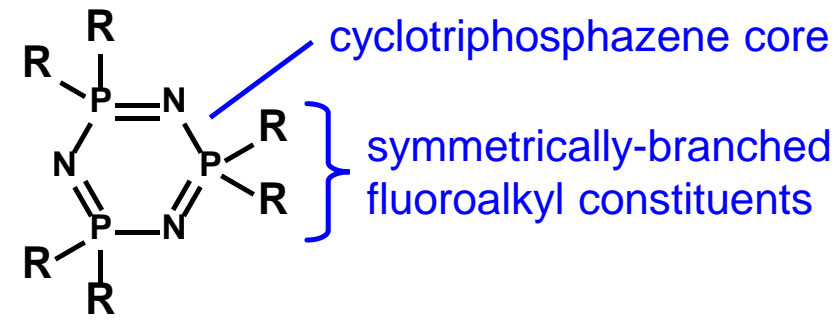

\begin{tabular}{|c|c|c|c|c|c|}
\hline Tune Mix Component & $\begin{array}{l}\text { Short } \\
\text { Name }\end{array}$ & R group & $\begin{array}{l}\text { Molecular } \\
\text { Formula }\end{array}$ & $\begin{array}{l}\text { lon } \\
\text { Form }\end{array}$ & $\begin{array}{c}\text { Exact lon } \\
\text { Mass } \\
\text { [Da] }\end{array}$ \\
\hline trimethylglycine & Betaine & none & $\mathrm{C}_{5} \mathrm{H}_{11} \mathrm{NO}_{2}$ & $+\mathrm{H}$ & 118.0863 \\
\hline \multirow{2}{*}{ hexamethoxyphosphazene } & \multirow{2}{*}{ HP-321 } & \multirow{2}{*}{$-\mathrm{OCH}_{3}$} & \multirow{2}{*}{$\mathrm{C}_{6} \mathrm{H}_{18} \mathrm{~N}_{3} \mathrm{O}_{6} \mathrm{P}_{3}$} & $+\mathrm{H}$ & 322.0481 \\
\hline & & & & $+\mathrm{Na}$ & 344.0301 \\
\hline \multirow{2}{*}{$\begin{array}{l}\text { hexakis(2,2-difluoroethoxy) } \\
\text { phosphazene }\end{array}$} & \multirow{2}{*}{ HP-621 } & \multirow{2}{*}{$-\mathrm{OCF}_{2} \mathrm{CHF}_{2}$} & \multirow{2}{*}{$\mathrm{C}_{12} \mathrm{H}_{18} \mathrm{~F}_{12} \mathrm{~N}_{3} \mathrm{O}_{6} \mathrm{P}_{3}$} & $+\mathrm{H}$ & 622.0290 \\
\hline & & & & $+\mathrm{Na}$ & 644.0109 \\
\hline \multirow{2}{*}{$\begin{array}{l}\text { hexakis(2,2,3,3-tetrafluoropropoxy) } \\
\text { phosphazene }\end{array}$} & \multirow{2}{*}{ HP-921 } & \multirow{2}{*}{$-\mathrm{OCH}_{2} \mathrm{CF}_{2} \mathrm{CHF}_{2}$} & \multirow{2}{*}{$\mathrm{C}_{18} \mathrm{H}_{18} \mathrm{~F}_{24} \mathrm{~N}_{3} \mathrm{O}_{6} \mathrm{P}_{3}$} & $+\mathrm{H}$ & 922.0098 \\
\hline & & & & $+\mathrm{Na}$ & 943.9917 \\
\hline \multirow{2}{*}{$\begin{array}{l}\text { hexakis(1h,1h,4h-hexafluorobutyloxy) } \\
\text { phosphazene }\end{array}$} & \multirow{2}{*}{ HP-1221 } & \multirow{2}{*}{$-\mathrm{OCH}_{2}\left(\mathrm{CF}_{2}\right)_{2} \mathrm{CHF}_{2}$} & \multirow{2}{*}{$\mathrm{C}_{24} \mathrm{H}_{18} \mathrm{~F}_{36} \mathrm{~N}_{3} \mathrm{O}_{6} \mathrm{P}_{3}$} & $+\mathrm{H}$ & 1221.9906 \\
\hline & & & & $+\mathrm{Na}$ & 1243.9726 \\
\hline \multirow{2}{*}{$\begin{array}{l}\text { hexakis(1h,1h,5h-octafluoropentoxy) } \\
\text { phosphazene }\end{array}$} & \multirow{2}{*}{ HP-1521 } & \multirow{2}{*}{$-\mathrm{OCH}_{2}\left(\mathrm{CF}_{2}\right)_{3} \mathrm{CHF}_{2}$} & \multirow{2}{*}{$\mathrm{C}_{30} \mathrm{H}_{18} \mathrm{~F}_{48} \mathrm{~N}_{3} \mathrm{O}_{6} \mathrm{P}_{3}$} & $+\mathrm{H}$ & 1521.9715 \\
\hline & & & & $+\mathrm{Na}$ & 1543.9534 \\
\hline
\end{tabular}

Figure S4. Components of the tuning mixture used in this work, which contains a low-mass component (betaine) and a series of symmetrically-branched cyclotriphosphazenes (hexyl-substituted phosphazenes, HP). ${ }^{5}$ The protonated ion form is predominantly observed in positive ion mode, however, sodium-adducted ion species can also be observed at low abundance. The nomenclature used here and in the main text utilizes "HP" followed by the neutral mass of the phosphazene (e.g., HP-321). 
Table S1. Chemical standards used in this work. Abbreviations used are listed in the left column.

\begin{tabular}{|c|c|c|c|}
\hline Analyte & Full Name & $\begin{array}{l}\text { Vendor Source } \\
\text { (Product Number) }\end{array}$ & $\begin{array}{l}\text { CAS Registry } \\
\text { Number }\end{array}$ \\
\hline Betaine & trimethylglycine & \multirow{6}{*}{$\begin{array}{l}\text { Agilent Technologies } \\
\text { (ESI-L, G1969-85000) }\end{array}$} & $107-43-7$ \\
\hline $\mathrm{HP}-321$ & hexamethoxyphosphazene & & $957-13-1$ \\
\hline $\mathrm{HP}-621$ & hexakis(2,2-difluoroethoxy) phosphazene & & $186817-57-2$ \\
\hline HP-921 & hexakis(2,2,3,3-tetrafluoropropoxy) phosphazene & & $58943-98-9$ \\
\hline HP-1221 & hexakis(1h,1h,4h-hexafluorobutyloxy) phosphazene & & $186406-47-3$ \\
\hline HP-1521 & hexakis(1h,1h,5h-octafluoropentoxy) phosphazene & & $16059-16-8$ \\
\hline SDGRG & H-Ser-Asp-Gly-Arg-Gly-OH & Sigma-Aldrich (S3771) & $108608-63-5$ \\
\hline GRGDS & H-Gly-Arg-Gly-Asp-Ser-OH & Sigma-Aldrich (G4391) & $96426-21-0$ \\
\hline GD1a & disialoganglioside $\mathrm{GD}_{1 \mathrm{a}}(\mathrm{d} 18: 1 / 18: 0)$ & Matreya, LLC (1062) & $12707-58-3$ \\
\hline GD1b & disialoganglioside $\mathrm{GD}_{1 \mathrm{~b}}(\mathrm{~d} 18: 1 / 18: 0)$ & Matreya, LLC (1501) & $19553-76-5$ \\
\hline LNFP1 & lacto-N-fucopentaose I & Carbosynth, LLC (OL05676) & $7578-25-8$ \\
\hline LNFP2 & lacto-N-fucopentaose II & Carbosynth, LLC (OL03876) & $21973-23-9$ \\
\hline 1-LG & 1-linoleoyl glycerol (18:2) & Cayman Chemical (10008869) & $2277-28-3$ \\
\hline 2-LG & 2-linoleoyl glycerol (18:2) & Cayman Chemical (62260) & $3443-82-1$ \\
\hline 1-AG & 1-arachidonoyl glycerol $(20: 4)$ & Cayman Chemical (62150) & $129691-05-0$ \\
\hline $2-A G$ & 2-arachidonoyl glycerol (20:4) & Cayman Chemical (62160) & $53847-30-6$ \\
\hline D-(+)-Melezitose & $\alpha$-D-glucosyl-(1,3)- $\beta$-D-fructosyl-(2,1)- $\alpha$-D-glucose & Alfa Aesar (AAB2220909) & 207511-10-2 \\
\hline 1-Kestose & $\beta$-D-fructosyl-(2,1)- $\beta$-D-fructosyl-(2,1)- $\alpha$-D-glucose & Sigma-Aldrich (72555) & $470-69-9$ \\
\hline D-(+)-Raffinose & $\alpha$-D-galactosyl-(1,6)- $\alpha$-D-glucosyl- $\beta$-D-fructose & Waters Corp. (700004768) & $512-69-6$ \\
\hline Isomaltotriose & $\alpha$-D-glucosyl-(1,6)- $\alpha$-D-glucosyl-(1,6)-D-glucose & Sigma-Aldrich (I0381) & $3371-50-4$ \\
\hline Maltotriose & $\alpha$-D-glucosyl-(1,4)- $\alpha$-D-glucosyl-(1,4)-D-glucose & Sigma-Aldrich (M8378) & $1109-28-0$ \\
\hline D-(+)-Cellotriose & $\beta$-D-glucosyl-(1,4)- $\beta$-D-glucosyl-(1,4)-D-glucose & Carbosynth, LLC (OC05719) & $33404-34-1$ \\
\hline
\end{tabular}


Table S2. Tabulated drift times, signal intensities, and signal-to-noise ratios for tune mix measured at various drift fields from 4.48 to $21.12 \mathrm{~V} / \mathrm{cm}$. Data is for single pulse (SP) and multiplexing (3- and 4-bit).

\begin{tabular}{|c|c|c|c|c|c|c|c|c|c|c|c|c|c|c|}
\hline \multirow{3}{*}{$\begin{array}{l}\text { Drift } \\
\text { Field } \\
\\
{[\mathrm{V} / \mathrm{cm}]}\end{array}$} & \multirow{3}{*}{ Analyte } & \multirow{3}{*}{ 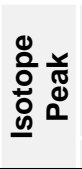 } & \multirow{3}{*}{$\begin{array}{c}\text { Exact } \\
\text { Ion } \\
\text { Mass } \\
\text { [Da] }\end{array}$} & \multirow{2}{*}{$\begin{array}{l}\text { Drift } \\
\text { Time } \\
\text { (DT) }\end{array}$} & \multicolumn{3}{|c|}{ Signal Intensity (SI) } & \multicolumn{3}{|c|}{$\begin{array}{c}\text { Signal-to-Noise } \\
\text { Ratio (S/N) }\end{array}$} & \multicolumn{2}{|c|}{$\begin{array}{c}\text { Change in } \\
\text { SI }\end{array}$} & \multicolumn{2}{|c|}{$\begin{array}{c}\text { Change in } \\
S / N\end{array}$} \\
\hline & & & & & SP & 3-bit & 4-bit & SP & 3-bit & 4-bit & $\begin{array}{l}\text { SP to } \\
\text { 3-bit }\end{array}$ & $\begin{array}{l}\text { SP to } \\
\text { 4-bit }\end{array}$ & $\begin{array}{l}\text { SP to } \\
\text { 3-bit }\end{array}$ & $\begin{array}{l}\text { SP to } \\
\text { 4-bit }\end{array}$ \\
\hline & & & & [ms] & \multicolumn{3}{|c|}{ [ion counts] } & [iol & $n$ coun & ts] & [fold $c$ & hange] & [fold c & lange \\
\hline 4.48 & $\mathrm{P}-321$ & $\bar{M}$ & 322.0481 & 63.68 & 0401 & 205372 & 474400 & 207 & 341 & 1014 & 3.40 & 7.85 & 2.61 & 4.90 \\
\hline & & $M+1$ & 323.0 & 63.69 & 4753 & 15916 & 36887 & 16 & 42 & 79 & & 7.76 & & 4.85 \\
\hline & & $M+2$ & 324.0524 & 63.70 & 925 & 3185 & 7352 & 3 & 8 & 16 & 3.44 & 7.95 & 2.65 & 4.96 \\
\hline & HP-621 & M & 622.0290 & 85.57 & 4634961 & 18862483 & 3592732 & 1587 & 4964 & 7680 & 4.07 & 7.75 & .13 & 4.84 \\
\hline & & $M+1$ & 623.0323 & 85.57 & 66631 & 258445 & 520285 & 228 & 680 & 1112 & 3.88 & 7.81 & 98 & 4.88 \\
\hline & & $M+2$ & 24.0332 & 85.60 & 9961 & 39439 & 69548 & 34 & 104 & 149 & 3.96 & 6.98 & .04 & 4.36 \\
\hline & HP-921 & M & 922.0098 & 102.95 & 23911 & 9055561 & 1838547 & 767 & 2383 & 3930 & 4.04 & 8.21 & 11 & 5.13 \\
\hline & & $M+1$ & 923.0132 & & & & & 157 & 485 & 819 & 4.01 & 8.33 & 08 & .20 \\
\hline & & $M+2$ & 924. & 102.91 & 7702 & 2 & 61029 & 26 & 80 & 130 & 3.97 & 7.92 & 55 & 95 \\
\hline & HP-1221 & $M$ & 1221.9906 & 121.35 & 80964 & 268794 & 580237 & 277 & 707 & 1240 & 3.32 & 7.17 & 55 & 47 \\
\hline & & $M+1$ & 1222.9940 & 121.35 & 21421 & 71758 & 154544 & 73 & 189 & 330 & 3.35 & 7.21 & 58 & 1.50 \\
\hline & & $M+2$ & 1223.9973 & 120.37 & 4060 & 12927 & 27309 & 14 & 34 & 58 & 3.18 & .73 & 45 & 4.20 \\
\hline & HP-1521 & M & 1521.9715 & 133.78 & 33686 & 91769 & 206159 & 115 & 242 & 441 & 2.72 & 0.12 & 09 & 3.82 \\
\hline & & $M+1$ & 1522 & & $10 s$ & 30 & & 38 & 30 & 147 & & .28 & 13 & 92 \\
\hline & & $M+2$ & 1523 & & & & & 7 & 18 & 31 & & 7.17 & 0 & 48 \\
\hline 5.76 & HP-321 & M & 1 & 5 & 3 & 1 & 678868 & 330 & 086 & 451 & 4.27 & 7.03 & 28 & 39 \\
\hline & & $M+1$ & 5 & 50.96 & 6 & 28 & 53943 & 22 & 75 & 115 & 4.46 & .44 & 13 & 27 \\
\hline & & $M+2$ & 24.0524 & 50.98 & 1205 & 5385 & 9982 & 4 & 14 & 21 & 4.47 & 3.28 & 44 & 17 \\
\hline & HP-621 & M & 22.0290 & 68.52 & 016922 & 25849784 & 4923406 & 402 & 803 & 10525 & 3.68 & 7.02 & .83 & 4.38 \\
\hline & & $M+1$ & 23 & 68.52 & 92701 & 371736 & 721202 & 317 & 978 & 1542 & 4.01 & 7.78 & 08 & 86 \\
\hline & & $M+2$ & 2 & 68.47 & & & & 51 & 41 & 237 & & 7.47 & 78 & 67 \\
\hline & HP-921 & M & & 8 & 349 & & & 195 & 76 & 5587 & & & & \\
\hline & & $M+$ & 2 & 82.65 & & 29 & 22 & 263 & 3 & 1180 & 3 & 7 & 1 & \\
\hline & & $M+2$ & 65 & 82.58 & 10745 & & 00 & 37 & 122 & 180 & 4 & 84 & 3 & 39 \\
\hline & $P-$ & M & 06 & 95.88 & & & 025 & 415 & 306 & 2044 & 4.09 & .88 & 15 & 92 \\
\hline & & $\mathrm{M}+$ & 1222. & 95.88 & 6 & & 256855 & 104 & 366 & 549 & 4.59 & 3.47 & 53 & 5.29 \\
\hline & & $M+2$ & 1223 & 95.96 & & & & 19 & 8 & 99 & & 45 & & 28 \\
\hline & HP-1521 & M & 1 & 10 & & & & 138 & 97 & 714 & & 30 & & 18 \\
\hline & & $M+1$ & 1 & & & & & 45 & 132 & 233 & & 8.39 & & \\
\hline & & $M+2$ & 1523 & 107 & 27 & & 37 & 9 & 26 & 47 & 3 & 8.00 & 80 & \\
\hline 7.04 & P-321 & M & 1 & 42.68 & 38025 & 562206 & 992824 & 473 & 480 & 122 & 4.07 & 9 & 13 & 9 \\
\hline & & $M+$ & & & & & & 34 & 116 & 176 & & 3.21 & 39 & \\
\hline & & $\mathrm{M}+$ & & 2 & & & 15204 & 7 & 21 & 33 & 4 & .96 & 17 & 97 \\
\hline & HP-621 & M & & & & & & 159 & 1 & 018 & & 61 & & \\
\hline & & $\mathrm{M}+$ & & & & & & 465 & & 2155 & & & & \\
\hline & & $M-$ & & & & & & 72 & & 325 & & 7.24 & & \\
\hline & HP-9LI & M & & ( & 11 & 189 & 71 & 1712 & 983 & 8559 & 3 & 8.00 & 91 & \\
\hline & & $M+$ & & 10 & & & 618 & 346 & 1050 & 739 & 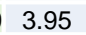 & 3.05 & .03 & \\
\hline & & $M+$ & & ( & & 2 & 57 & 52 & 57 & 269 & 3 & & & \\
\hline & $\perp$ & M & & 2 & & & & 563 & 1777 & 039 & & .65 & 6 & 10 \\
\hline & & $\mathrm{M}+$ & & & & & & 160 & 418 & 713 & & 15 & & \\
\hline & & $\mathrm{M}+$ & & & & & & 28 & 1 & 139 & & & & \\
\hline & r & M & & & & & & 158 & & 799 & & & & \\
\hline & & $\mathrm{M}+$ & & & & & 12 & 55 & 168 & 258 & 3 & 7.49 & .05 & \\
\hline & & $\mathrm{N}$ & & & & 2 & 32 & 12 & 33 & 50 & 3 & .97 & .88 & \\
\hline 8.3 & 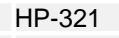 & M & & 97 & 26 & 708 & 284 & 600 & 1864 & 2953 & 4 & 38 & 11 & \\
\hline & & $M+1$ & & 37.03 & & & & 43 & 144 & 217 & 4.31 & 7.99 & 32 & 99 \\
\hline & & $M+2$ & & 37.12 & 3120 & 27 & & 11 & 30 & 47 & 3.69 & 6.99 & 84 & 36 \\
\hline & HP-621 & M & & & & & & 536 & & 921 & 3. & 7. & & \\
\hline & & & & & & & & 541 & 1 & 2761 & & & & \\
\hline & & $M+$ & & & & & & 90 & 55 & 466 & 0 & & 35 & \\
\hline & 1 & $\mid \mathrm{IV}$ & & 60 & & & 12 & 2192 & 200 & 10692 & 3 & & 83 & \\
\hline & & $M+1$ & & 60.12 & 74 & 489667 & 1068468 & 469 & 1289 & 2284 & 3.57 & 7.79 & .75 & 4.87 \\
\hline & & $M+2$ & 65 & 60.13 & 243 & 69861 & 149775 & 62 & 184 & 320 & 3.83 & 8.21 & .94 & 5.13 \\
\hline & HP-1221 & M & 1221 & 69.79 & 32 & & 915 & 762 & 2357 & 3634 & 4.0 & 7.6 & 09 & 4.77 \\
\hline & & $M+$ & & & & & & 193 & & 917 & & & & \\
\hline & & $\mathrm{M}+$ & & & & & & 36 & 09 & 168 & & 1 & 03 & \\
\hline & & M & & & & & & 208 & 15 & 056 & 4.47 & 14 & 44 & 08 \\
\hline & & $\mathrm{M}+$ & & & & & 152858 & 63 & 193 & 327 & 4.01 & 8.37 & 3.08 & 5.22 \\
\hline & & $M+2$ & 1523.9781 & 78.44 & 4025 & 14894 & 31413 & 14 & 39 & 67 & 3.70 & 7.80 & 2.84 & 4.87 \\
\hline
\end{tabular}


Table S2. Continued

\begin{tabular}{|c|c|c|c|c|c|c|c|c|c|c|c|c|c|c|}
\hline \multirow{3}{*}{$\begin{array}{l}\text { Drift } \\
\text { Field } \\
{[\mathrm{V} / \mathrm{cm}]}\end{array}$} & \multirow{3}{*}{ Analyte } & \multirow{3}{*}{ 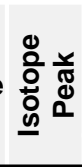 } & \multirow{3}{*}{$\begin{array}{c}\text { Exact } \\
\text { Ion } \\
\text { Mass } \\
\text { [Da] }\end{array}$} & \multirow{3}{*}{$\begin{array}{c}\text { Drift } \\
\text { Time } \\
\text { (DT) } \\
\text { [ms] }\end{array}$} & \multicolumn{3}{|c|}{ Signal Intensity (SI) } & \multicolumn{3}{|c|}{$\begin{array}{c}\text { Signal-to-Noise } \\
\text { Ratio (S/N) }\end{array}$} & \multicolumn{2}{|c|}{$\begin{array}{c}\text { Change in } \\
\text { SI }\end{array}$} & \multicolumn{2}{|c|}{$\begin{array}{l}\text { Change in } \\
S / N\end{array}$} \\
\hline & & & & & SP & 3-bit & 4-bit & SP & 3-bit & 4-bit & $\begin{array}{l}\text { SP to } \\
\text { 3-bit }\end{array}$ & $\begin{array}{l}\text { SP to } \\
\text { 4-bit }\end{array}$ & $\begin{array}{l}\text { SP to } \\
\text { 3-bit }\end{array}$ & $\begin{array}{l}\text { SP to } \\
\text { 4-bit }\end{array}$ \\
\hline & & & & & \multicolumn{3}{|c|}{ [ion counts] } & [ior & $n$ coun & nts] & & & & hang \\
\hline & HP-321 & $\bar{M}$ & 322.0481 & 32.73 & 218256 & 846411 & 1660184 & 747 & $2<<0$ & $3 \quad 3549$ & 3.88 & 7.61 & 2.98 & 4.75 \\
\hline & & $M+1$ & 323.0515 & 2.73 & 15803 & 55835 & & 54 & 147 & 261 & & & & 82 \\
\hline & & $M+2$ & & .82 & & & & 10 & 30 & 51 & & & & 95 \\
\hline & HP-621 & M & 90 & & 78308 & 345482 & 725300 & 5403 & 701 & 065 & 4.02 & 3 & 09 & 64 \\
\hline & & $\mathrm{M}+$ & 23 & & & & & 624 & & 151 & 4.08 & & 13 & 05 \\
\hline & & $M+2$ & 32 & & & 8 & & 97 & 280 & 442 & 3.74 & & 87 & .53 \\
\hline & HP-921 & M & 98 & .25 & & 73 & 89 & 2711 & 8173 & 313608 & 3.92 & & & .02 \\
\hline & & $M+1$ & 32 & .25 & & & & 550 & 1612 & 2690 & 3.82 & & & 89 \\
\hline & & $M+2$ & 924.0165 & & & & & 75 & 237 & 386 & & & 16 & 15 \\
\hline & HP-1221 & M & & & & 33 & & 921 & 2862 & 2. 4854 & & & 11 & .27 \\
\hline & & $M+1$ & 40 & & & 2 & & 241 & 773 & 3 1223 & & & & .07 \\
\hline & & $M+2$ & 73 & & & & & 43 & 121 & L 196 & & & & \\
\hline & HP-1 & M & 15 & 2 & & 339 & & 268 & 893 & $3 \quad 1326$ & 4.34 & & 34 & 95 \\
\hline & & $M+1$ & & & & & & 71 & 229 & 374 & 4.20 & & 23 & 5.27 \\
\hline & & $M+2$ & & & & & & 18 & 49 & 80 & & & & 55 \\
\hline .88 & HP-321 & M & & 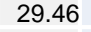 & 0 & 1 & & 883 & 615 & 3963 & & & & 49 \\
\hline & & $M+1$ & & 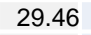 & & 6 & & 75 & 175 & 325 & 3. & & 34 & 36 \\
\hline & & $M+2$ & & 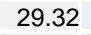 & & 1 & & 11 & 34 & 53 & 4.03 & & & .86 \\
\hline & HP-621 & M & & 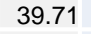 & 1818 & 2732 & & 6227 & 143 & 28641 & & & & 60 \\
\hline & & $\mathrm{M}+$ & & & & & & 699 & & 3123 & & & & 47 \\
\hline & & $\mathrm{M}+$ & & & & & & 115 & 327 & 557 & & & & .85 \\
\hline & $\mathrm{H}$ & M & & & & 02 & & 3185 & 536 & 15816 & & & & 97 \\
\hline & & $M+$ & & & & & & 645 & 55 & 2868 & & & & 45 \\
\hline & & $M+2$ & & & & & & 88 & 241 & 425 & & & & 83 \\
\hline & HP-1221 & M & & & & & & 1108 & 3435 & 5410 & & & & .88 \\
\hline & & $\mathrm{M}+$ & & & & & & 239 & 696 & 1145 & & & & .79 \\
\hline & & $M+$ & & & & & & 52 & 33 & 3260 & & & & \\
\hline & HP-1521 & M & & & & & & 320 & 1058 & 3 1702 & & & & 33 \\
\hline & & $\mathrm{M}+$ & & & & & & 83 & 275 & 433 & & & & 5.21 \\
\hline & & $\mathrm{M}+$ & & & & & & 17 & 44 & 85 & & & & .08 \\
\hline 16 & HP-321 & M & & & & 9 & & 860 & 2669 & 4319 & & & & .02 \\
\hline & & $M+$ & & & & & & 78 & 228 & $3 \quad 355$ & 3 & & & \\
\hline & & $\mathrm{M}+$ & & & & & & 12 & 36 & 58 & & & & 80 \\
\hline & HP-621 & M & & & & 1. & & 010 & 88 & 184 & 3. & & & .59 \\
\hline & & $\mathrm{M}+$ & & & & & & 834 & & +3905 & & & & 68 \\
\hline & & $\mathrm{M}+$ & & & & & & 132 & 409 & 650 & 4 & & & 93 \\
\hline & HIP-9L & M & & & & & & 3723 & 10919 & 18316 & 3. & & & 32 \\
\hline & & $M-$ & & & & & & 744 & 1862 & 3206 & 3. & & 50 & 4.31 \\
\hline & & $M+2$ & & & & & & 100 & 282 & 431 & & & & 1.33 \\
\hline & HP-1221 & M & & & & & & 1298 & & 295 & & & & \\
\hline & & $\mathrm{M}+$ & & & & & & 286 & 20 & 112 & & & & \\
\hline & & $\mathrm{M}+$ & & & & & & 44 & 20 & $t$ & & & & \\
\hline & + & M & & & & & & 343 & 1215 & 1741 & & & & 07 \\
\hline & & $\mathrm{M}+$ & & & & & & 94 & 304 & 468 & 4. & & & 99 \\
\hline & & $M+$ & & & & & & 21 & 52 & 102 & 3. & & & \\
\hline 13.44 & HP-32 & M & & & & & & 1157 & 3196 & 4848 & & & & \\
\hline & & & & & & & & 80 & 34 & 38 & & & & \\
\hline & & $\mathrm{M}+$ & & & & 22 & & 10 & 31 & 49 & 3. & & 1 & 1.76 \\
\hline & HP-621 & M & & & & 27 & & 7553 & 23033 & 750 & 3. & & 5 & 60 \\
\hline & & $\mathrm{M}+$ & & & & & & 824 & 2604 & 3661 & & & & \\
\hline & & M & & & & & & & 99 & 646 & & & & \\
\hline & HP-921 & M & & & & & & 40 & & 14 & & & & \\
\hline & & & & & & & & 806 & 2022 & 3503 & 3. & & & 1.35 \\
\hline & & $M+2$ & & & & & & 106 & 297 & 498 & 3.65 & & 81 & 4.70 \\
\hline & HP-1221 & M & & 46 & & & & 1431 & 4229 & 7501 & 3. & & 66 & 24 \\
\hline & & $M-$ & & & & & & 331 & 20 & 149 & & & & \\
\hline & & & & & & & & 68 & & & & & & \\
\hline & & M & & & & & & 421 & 1353 & 2016 & 4.18 & 7.67 & 21 & .19 \\
\hline & & $M+1$ & 522.9748 & 52.70 & & 136514 & & 116 & 359 & 539 & 4.03 & 7.43 & 3.10 & 4.64 \\
\hline & & $M+2$ & 1523.9781 & 52.67 & 6129 & 21542 & 49587 & $\angle \perp$ & 57 & 106 & 3.51 & 8.09 & 2.70 & 5.05 \\
\hline
\end{tabular}


Table S2. Continued

\begin{tabular}{|c|c|c|c|c|c|c|c|c|c|c|c|c|c|c|}
\hline \multirow{3}{*}{$\begin{array}{l}\text { Drift } \\
\text { Field } \\
{[\mathrm{V} / \mathrm{cm}]}\end{array}$} & \multirow{3}{*}{ Analyte } & \multirow{3}{*}{ 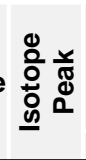 } & \multirow{3}{*}{$\begin{array}{c}\text { Exact } \\
\text { lon } \\
\text { Mass } \\
\text { [Da] }\end{array}$} & \multirow{2}{*}{$\begin{array}{c}\text { Drift } \\
\text { Time } \\
\text { (DT) }\end{array}$} & \multicolumn{3}{|c|}{ Signal Intensity (SI) } & \multicolumn{3}{|c|}{$\begin{array}{c}\text { Signal-to-Noise } \\
\text { Ratio (S/N) }\end{array}$} & \multicolumn{2}{|c|}{$\begin{array}{c}\text { Change in } \\
\text { SI }\end{array}$} & \multicolumn{2}{|c|}{$\begin{array}{c}\text { Change in } \\
S / N\end{array}$} \\
\hline & & & & & SP & 3-bit & 4-bit & SP & 3-bit & 4-bit & $\begin{array}{l}\text { SP to } \\
\text { 3-bit }\end{array}$ & $\begin{array}{l}\text { SP to } \\
\text { 4-bit }\end{array}$ & $\begin{array}{l}\text { SP to } \\
\text { 3-bit }\end{array}$ & $\begin{array}{l}\text { SP to } \\
\text { 4-bit }\end{array}$ \\
\hline & & & & [ms] & \multicolumn{3}{|c|}{ [ion counts] } & [ior & n coun & & [fold c & hange] & fold c & hang \\
\hline 14.72 & HP-321 & $M$ & 322.0481 & 23.01 & 332570 & 1236996 & 2377489 & 1139 & 3256 & 5082 & 3.72 & 7.15 & 2.86 & 4.46 \\
\hline & & $M+1$ & 323.0515 & 23.03 & 24024 & 75635 & 164183 & 82 & 199 & 351 & 3.15 & 6.83 & 2.42 & 4.27 \\
\hline & & $M+2$ & & 3.11 & 5058 & & & 17 & 47 & 71 & 3.52 & 53 & & 4.08 \\
\hline & HP-621 & M & 622.0290 & 31.032 & 2427149 & 92190781 & 17243702 & 8309 & 24264 & 36861 & 3.80 & 7.10 & 2.92 & 4.44 \\
\hline & & $M+1$ & 623. & 31.03 & 261210 & 1047219 & 1799465 & 894 & 2756 & 3847 & 4.01 & 6.89 & 3.08 & 4.30 \\
\hline & & $M+2$ & 624.0332 & 31.04 & 45072 & 169901 & 312759 & 154 & 447 & 669 & 3.77 & 94 & 2.90 & 4.33 \\
\hline & HP-921 & M & 922.0098 & 37.48 & 1300354 & 5006551 & 10018413 & 4452 & 13177 & 21416 & 3.85 & .70 & 2.96 & 4.81 \\
\hline & & $M+1$ & 923.0132 & 37.48 & 252057 & 560 & 1727279 & 863 & 2131 & 3692 & 3.21 & 85 & 47 & 4.28 \\
\hline & & $M+2$ & 924.0165 & 37.48 & 38404 & 128708 & 259243 & 131 & 339 & 554 & 3.35 & 75 & .58 & 4.22 \\
\hline & HP-1221 & M & 1221.9906 & 43.53 & 467369 & 1816 & 3820353 & 1600 & 4781 & 8167 & 3.89 & 17 & 2.99 & 5.10 \\
\hline & & $M+1$ & 222. & 3.53 & 87472 & & & 299 & 913 & 1410 & 3.97 & 54 & 3.05 & 4.71 \\
\hline & & $M+2$ & 973 & 3.54 & 16160 & 60 & & 55 & 148 & 274 & & & 67 & 4.95 \\
\hline & HP-1521 & M & 1521 & 98 & 127 & & & 435 & 1466 & 2279 & 4.38 & 38 & 3.37 & .24 \\
\hline & & $M+1$ & 48 & 8.98 & 33294 & & & 114 & 350 & 533 & 4.00 & 49 & 3.07 & .68 \\
\hline & & $M+2$ & 781 & 8.96 & 6424 & 28 & 38 & 22 & 61 & 110 & 3.62 & 02 & 2.78 & .01 \\
\hline 16.00 & HP-321 & M & 481 & 1.54 & 372891 & 126 & & 1277 & 3332 & 5163 & 3.40 & 48 & 61 & 4.04 \\
\hline & & $M+1$ & 15 & 1.50 & 30589 & & & 105 & 269 & 400 & & 11 & .56 & 3.81 \\
\hline & & $\mathrm{M}+$ & 24 & 21.41 & 5058 & 17820 & 3045 & 17 & 47 & 71 & 3.52 & & 71 & .08 \\
\hline & IP_62 & M & 90 & 29.05 & 2553648 & 812401 & 17895733 & 742 & 25826 & 38255 & 3.84 & .01 & 2.95 & 4.38 \\
\hline & & $M+1$ & 623.0323 & 29.05 & 265290 & 1096928 & 1870915 & 908 & 2887 & 3999 & 4.13 & 05 & 3.18 & 4.40 \\
\hline & & $M+2$ & & 29.05 & & & & 158 & 89 & 723 & & & 08 & .56 \\
\hline & $F$ & M & & 35.08 & 58 & 65 & 220 & 4841 & 14148 & 22630 & 3.80 & & .92 & 1.67 \\
\hline & & $M+1$ & 32 & 35.08 & 256628 & 848413 & 1802830 & 879 & 2233 & 3854 & 3.31 & 3 & & 4.39 \\
\hline & & $M+2$ & & 19 & & & & 134 & 58 & 564 & & & 66 & 4.20 \\
\hline & & M & & 4 & & & & 1743 & 5077 & 8608 & & & & 4.94 \\
\hline & & $M+1$ & 40 & .74 & & & & 484 & 1443 & 2314 & 3.88 & 66 & & 4.78 \\
\hline & & $M+2$ & 1223.9973 & 40.76 & 26993 & & & 92 & 38 & 438 & & & & 4.74 \\
\hline & HP-1521 & M & & 2 & 136 & & & 468 & 1627 & 2378 & & & & .08 \\
\hline & & $\mathrm{M}+$ & & .84 & & & & 115 & 377 & 548 & & & & 4.76 \\
\hline & & $M+2$ & 31 & 5.81 & 344 & & & 25 & 65 & 119 & & 56 & & 4.72 \\
\hline 17.28 & HP-321 & M & & 2 & & & & 1415 & 3615 & 5562 & & & & 3.93 \\
\hline & & $M+$ & & 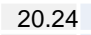 & & & & 118 & (37) & 436 & & & & 69 \\
\hline & & $\mathrm{M}+$ & & 0.21 & 6270 & 19754 & 3541 & 21 & 52 & 82 & 3.15 & 5 & 42 & 3.84 \\
\hline & HP-621 & M & & 27.342 & & & & 9145 & 260 & 40044 & 3. & & & 4.38 \\
\hline & & $\mathrm{M}+$ & & 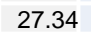 & & & & 1014 & 20 & 4167 & & & & 4.11 \\
\hline & & $M+$ & & & & & & 163 & 69 & 771 & 3.74 & 57 & 88 & .73 \\
\hline & HP-921 & M & & $33.02]$ & 147 & & 112 & 5066 & 14738 & 23995 & 3 & & 1 & 4.74 \\
\hline & & $M+$ & & & & & & 938 & & 4099 & & & & .37 \\
\hline & & $M+$ & & & & & & 129 & 85 & 605 & & & & .70 \\
\hline & 1 & M & & & & & & 1828 & 43 & 9263 & & 11 & 2 & 5.07 \\
\hline & & $M+$ & & & & & & 394 & & 1724 & & & & 37 \\
\hline & & $M+$ & & & & & & 8 & 25 & 383 & & & & 63 \\
\hline & HP-1521 & M & & .13 & 14 & 77 & 11 & 486 & 1652 & 2511 & 4.42 & 7 & 10 & 5.17 \\
\hline & & $M+$ & & & & & & 125 & 425 & 652 & & & & 5.20 \\
\hline & & $M+$ & & & & & & 32 & 97 & 0 & & & & 70 \\
\hline 18.56 & HP-321 & M & & & 8 & 0 & & 1501 & 3641 & 5640 & & & & 3.76 \\
\hline & & $\mathrm{M}+$ & & 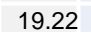 & & & & 86 & 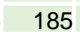 & 347 & & & & \\
\hline & & $M+$ & & 19. & 6270 & & & 21 & 52 & 82 & 3 & & & \\
\hline & $\mathrm{H}$ & M & & & & 9 & & 9499 & 27048 & 40206 & 3. & & & 4.23 \\
\hline & & $\mathrm{M}+$ & & 37 & 283 & 1140373 & & 972 & 3001 & 4199 & 4.02 & 2 & 99 & 4.32 \\
\hline & & $M+$ & & & & & & 183 & & 768 & 3. & & & 4.19 \\
\hline & & M & & & & & & 5290 & 15529 & 24827 & & & & 69 \\
\hline & & $\mathrm{M}+$ & & 24 & & & 19 & 946 & 2472 & 4272 & 3.40 & 7.23 & 61 & 4.51 \\
\hline & & $M+2$ & & & & & & 140 & & 12 & & & & 4.37 \\
\hline & HP-1221 & M & & & & & & 1605 & & 7039 & & & & (3) \\
\hline & & $M-$ & & & & & & 340 & 1051 & 1532 & 4.01 & & 09 & 4.50 \\
\hline & & $\mathrm{M}+$ & & 5 & & & & 68 & 173 & 324 & 3.29 & 7.58 & 53 & 4.73 \\
\hline & I & M & & & & & & 516 & 1616 & 2383 & 4.07 & & 10 & 4.62 \\
\hline & & $M-$ & & 40 & & & & 128 & 418 & 630 & 4.25 & 7.88 & 3.26 & 4.92 \\
\hline & & $M+$ & 3.9781 & 40.81 & 8861 & 28463 & 63228 & 30 & 75 & 135 & 3.21 & 7.14 & 2.47 & 4.46 \\
\hline
\end{tabular}

Table S2. Continued 


\begin{tabular}{|c|c|c|c|c|c|c|c|c|c|c|c|c|c|c|}
\hline \multirow{3}{*}{$\begin{array}{l}\text { Drift } \\
\text { Field } \\
{[\mathrm{V} / \mathrm{cm}]}\end{array}$} & \multirow{3}{*}{ Analyte } & \multirow{3}{*}{ 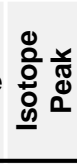 } & \multirow{3}{*}{$\begin{array}{c}\text { Exact } \\
\text { Ion } \\
\text { Mass } \\
\text { [Da] }\end{array}$} & \multirow{3}{*}{$\begin{array}{c}\text { Drift } \\
\text { Time } \\
\text { (DT) } \\
\text { [ms] }\end{array}$} & \multicolumn{3}{|c|}{ Signal Intensity (SI) } & \multicolumn{3}{|c|}{$\begin{array}{l}\text { Signal-to-Noise } \\
\text { Ratio (S/N) }\end{array}$} & \multicolumn{2}{|c|}{$\begin{array}{c}\text { Change in } \\
\text { SI }\end{array}$} & \multicolumn{2}{|c|}{$\begin{array}{c}\text { Change in } \\
S / N\end{array}$} \\
\hline & & & & & SP & 3-bit & 4-bit & SP & 3-bit & 4-bit & $\begin{array}{l}\text { SP to } \\
\text { 3-bit }\end{array}$ & $\begin{array}{l}\text { SP to } \\
\text { 4-bit }\end{array}$ & $\begin{array}{l}\text { SP to } \\
\text { 3-bit }\end{array}$ & $\begin{array}{l}\text { SP to } \\
\text { 4-bit }\end{array}$ \\
\hline & & & & & \multicolumn{3}{|c|}{ [ion counts] } & \multicolumn{3}{|c|}{ [ion counts] } & \multicolumn{4}{|c|}{ [fold change] [fold change] } \\
\hline \multirow[t]{15}{*}{19.84} & HP-321 & $M$ & 322.0481 & 18.26 & 7503 & 1454421 & 5283 & 1532 & 3828 & 5405 & 3.25 & 65 & 2.50 & 3.53 \\
\hline & & $M+1$ & 515 & 4 & 25572 & & & 88 & 214 & 320 & & & & .66 \\
\hline & & $M+2$ & 24 & .19 & 4587 & 15645 & 19 & 16 & 41 & 61 & & 22 & & 89 \\
\hline & HP-621 & M & 90 & 24.58 & 392829 & 10273285 & 036863 & 904 & 27039 & 40694 & & 58 & & 11 \\
\hline & & $M+1$ & 3.0323 & 24.58 & 297391 & 1036836 & 1982012 & 1018 & 2729 & 4237 & 3. & 6 & 8 & .16 \\
\hline & & $M+2$ & 4.0332 & 24.58 & 56403 & 98675 & 2746 & 193 & 523 & 797 & 3. & & & 4.13 \\
\hline & 92 & M & 2.0098 & 29.68 & 1644217 & 6184429 & 12023662 & 5629 & 16277 & 25703 & 3. & & 99 & 4.57 \\
\hline & & $M+1$ & 32 & 29.68 & & & & 1009 & 2506 & 4503 & & & & 4.46 \\
\hline & & $M+2$ & 924. & 9 & & & & 51 & 97 & 44 & & & & 4.26 \\
\hline & HP-1221 & M & & & & & & & 5841 & 344 & & & & 86 \\
\hline & & $M+1$ & & & & & & 5 & 73 & 59 & & & & \\
\hline & & $M+2$ & & & & & & 1 & 75 & 326 & & & & 62 \\
\hline & HP-1521 & M & & & & & & 0 & 1886 & 949 & & & & \\
\hline & & $M+1$ & & & & & & 1 & 453 & 72 & & & & 4.89 \\
\hline & & $M+2$ & 1 & is & 7 & & $t$ & 31 & 86 & 138 & 3. & & & 53 \\
\hline \multirow[t]{15}{*}{21.12} & 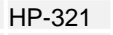 & M & 481 & 17.42 & 9033 & 035469 & 000066 & 161 & 2725 & 4275 & 3. & 0 & 5 & .68 \\
\hline & & $M+1$ & & 17. & 15476 & 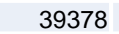 & 0 & 53 & 104 & 202 & 2. & & & 80 \\
\hline & & $M+2$ & 4 & 17.34 & 4215 & 12589 & 2522 & 14 & 33 & 48 & 2. & & 0 & 34 \\
\hline & HP-621 & M & 290 & 23.45 & 63953 & 556079 & 19500344 & 0147 & 27783 & 41685 & 3.5 & & 4 & .11 \\
\hline & & $M+1$ & & 23.45 & 292347.4 & 1082605 & 201 & 1001 & 2849 & 4305 & 3.7 & & 5 & 4.30 \\
\hline & & $M+2$ & 332 & 23.46 & 5688 & 3816 & 2808 & 195 & 536 & 840 & 3.58 & & 75 & 4.31 \\
\hline & St & M & & 28.32 & 1698821 & 6267293 & 12246107 & 5816 & 16495 & 26178 & 3. & & 34 & 4.50 \\
\hline & & $M+$ & & & & & & 989 & 2455 & 4332 & & & & 4.38 \\
\hline & & $M+2$ & & 28.32 & 5 & & & 149 & 23 & 650 & 3. & & & 4.36 \\
\hline & & M & & & 5858 & & & 2006 & 12 & 577 & & & & \\
\hline & & $M+$ & & & & & & 348 & 114 & 604 & 4.1 & & & \\
\hline & & $M+2$ & & & & & & 73 & 193 & 334 & 3.44 & & 84 & 4.58 \\
\hline & & M & & & & & & & 23 & 2948 & & & & 89 \\
\hline & & & & & & & & 1 & 397 & 600 & 3. & & 22 & t.5/ \\
\hline & & $M+2$ & 1523.9781 & 37.01 & 9285 & 29376 & 65321 & 32 & 77 & 140 & 3.16 & 7.04 & 2.43 & 4.39 \\
\hline
\end{tabular}




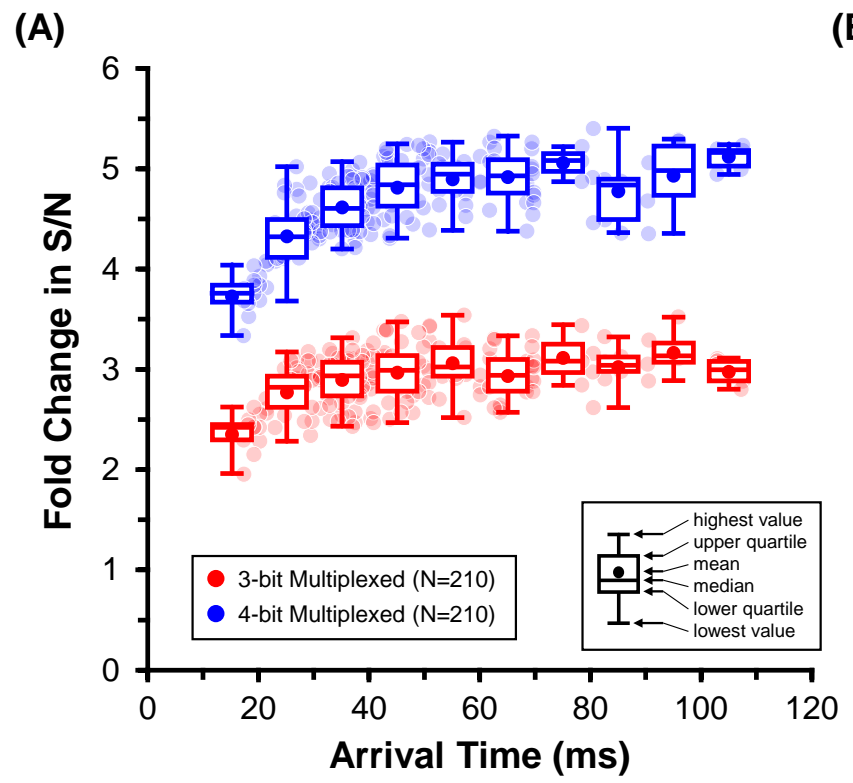

(B)

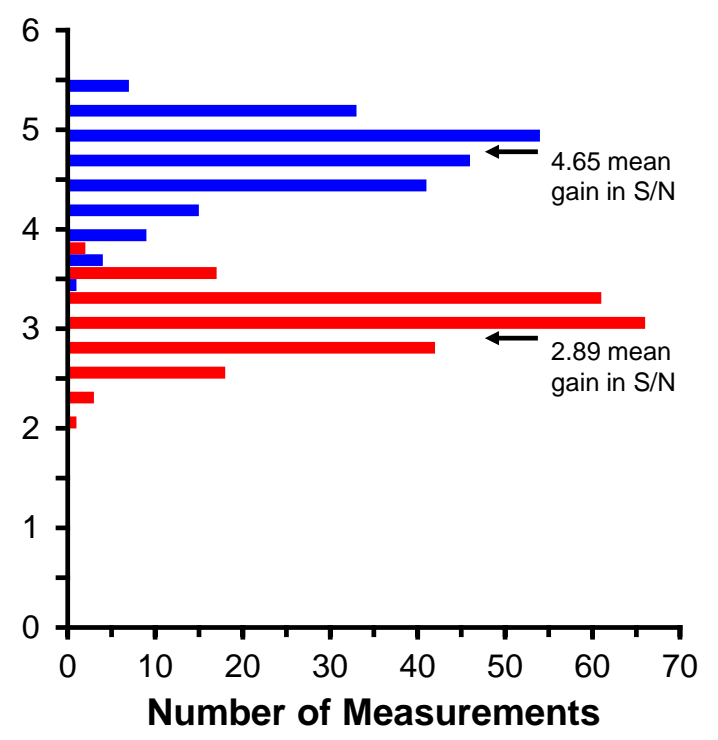

Figure S5. (A) Box-and-whisker plot of drift time versus the fold change in the signal-to-noise ratio $(\mathrm{S} / \mathrm{N})$ for the tune mix at various drift fields. Data for both 3- and 4-bit multiplexing are shown and compared to data obtained in single pulse mode. (B) Histograms indicating the distribution of measurements integrated across all ion mobility arrival times. 
Table S3. Fold change in signal intensity (SI).

\begin{tabular}{|c|c|c|c|c|c|c|c|c|c|c|c|c|c|c|}
\hline \multirow[b]{2}{*}{ Box \# } & \multirow[b]{2}{*}{ DT Range } & \multirow[b]{2}{*}{$\mathbf{N}$} & \multicolumn{6}{|c|}{ Fold Change in SI from SP to 3-bit } & \multicolumn{6}{|c|}{ Fold Change in Intensity from SP to 4-bit } \\
\hline & & & MIN & MAX & MEAN & MEDIAN & Q1 & Q3 & MIN & MAX & MEAN & MEDIAN & Q1 & Q3 \\
\hline 1 & $10-19$ & 9 & 2.54 & 3.41 & 3.06 & 3.15 & 2.99 & 3.18 & 5.34 & 6.46 & 5.97 & 6.02 & 5.86 & 6.15 \\
\hline 2 & $20-29$ & 39 & 2.97 & 4.13 & 3.61 & 3.68 & 3.40 & 3.82 & 5.91 & 8.04 & 6.94 & 6.92 & 6.60 & 7.20 \\
\hline 3 & $30-39$ & 48 & 3.16 & 4.31 & 3.77 & 3.82 & 3.55 & 4.00 & 6.73 & 8.11 & 7.39 & 7.37 & 7.10 & 7.71 \\
\hline 4 & $40-49$ & 39 & 3.21 & 4.52 & 3.85 & 3.88 & 3.62 & 4.08 & 6.90 & 8.40 & 7.72 & 7.74 & 7.41 & 8.06 \\
\hline 5 & $50-59$ & 21 & 3.28 & 4.61 & 3.98 & 3.94 & 3.82 & 4.18 & 7.03 & 8.44 & 7.85 & 7.91 & 7.67 & 8.09 \\
\hline 6 & $60-69$ & 24 & 3.35 & 4.34 & 3.83 & 3.81 & 3.61 & 4.03 & 7.02 & 8.53 & 7.89 & 7.89 & 7.62 & 8.15 \\
\hline 7 & $70-79$ & 3 & 3.70 & 4.47 & 4.06 & 4.01 & 3.86 & 4.24 & 7.80 & 8.37 & 8.10 & 8.14 & 7.97 & 8.25 \\
\hline 8 & $80-89$ & 9 & 3.41 & 4.33 & 3.93 & 3.96 & 3.87 & 4.07 & 6.98 & 8.65 & 7.64 & 7.75 & 7.20 & 7.84 \\
\hline 9 & $90-99$ & 6 & 3.75 & 4.59 & 4.12 & 4.07 & 3.99 & 4.23 & 6.97 & 8.47 & 7.90 & 8.00 & 7.59 & 8.37 \\
\hline 10 & $100-109$ & 6 & 3.64 & 4.04 & 3.88 & 3.91 & 3.77 & 4.00 & 7.92 & 8.39 & 8.19 & 8.26 & 8.05 & 8.32 \\
\hline
\end{tabular}

Table S4. Fold change in signal-to-noise ratio (S/N).

\begin{tabular}{|c|c|c|c|c|c|c|c|c|c|c|c|c|c|c|}
\hline \multirow[b]{2}{*}{ Box \# } & \multirow[b]{2}{*}{ DT Range } & \multirow[b]{2}{*}{$\mathbf{N}$} & \multicolumn{6}{|c|}{ Fold Change in S/N from SP to 3-bit } & \multicolumn{6}{|c|}{ Fold Change in S/N from SP to 4-bit } \\
\hline & & & MIN & MAX & MEAN & MEDIAN & Q1 & Q3 & MIN & MAX & MEAN & MEDIAN & Q1 & Q3 \\
\hline 1 & $10-19$ & 9 & 1.96 & 2.62 & 2.35 & 2.42 & 2.30 & 2.45 & 3.34 & 4.03 & 3.72 & 3.76 & 3.66 & 3.84 \\
\hline 2 & $20-29$ & 39 & 2.28 & 3.18 & 2.78 & 2.83 & 2.62 & 2.93 & 3.69 & 5.02 & 4.33 & 4.32 & 4.12 & 4.50 \\
\hline 3 & $30-39$ & 48 & 2.43 & 3.32 & 2.90 & 2.94 & 2.73 & 3.07 & 4.20 & 5.07 & 4.62 & 4.60 & 4.44 & 4.82 \\
\hline 4 & $40-49$ & 39 & 2.47 & 3.48 & 2.96 & 2.98 & 2.78 & 3.13 & 4.31 & 5.24 & 4.82 & 4.83 & 4.63 & 5.03 \\
\hline 5 & $50-59$ & 21 & 2.52 & 3.54 & 3.06 & 3.03 & 2.93 & 3.22 & 4.39 & 5.27 & 4.90 & 4.94 & 4.79 & 5.05 \\
\hline 6 & $60-69$ & 24 & 2.57 & 3.34 & 2.95 & 2.93 & 2.78 & 3.10 & 4.38 & 5.33 & 4.92 & 4.93 & 4.76 & 5.09 \\
\hline 7 & $70-79$ & 3 & 2.84 & 3.44 & 3.12 & 3.08 & 2.96 & 3.26 & 4.87 & 5.22 & 5.06 & 5.08 & 4.98 & 5.15 \\
\hline 8 & $80-89$ & 9 & 2.62 & 3.33 & 3.02 & 3.04 & 2.97 & 3.13 & 4.36 & 5.40 & 4.77 & 4.84 & 4.49 & 4.89 \\
\hline 9 & $90-99$ & 6 & 2.88 & 3.53 & 3.17 & 3.13 & 3.07 & 3.26 & 4.35 & 5.29 & 4.93 & 4.99 & 4.74 & 5.22 \\
\hline 10 & 100-109 & 6 & 2.80 & 3.11 & 2.98 & 3.00 & 2.90 & 3.08 & 4.95 & 5.24 & 5.12 & 5.16 & 5.03 & 5.20 \\
\hline
\end{tabular}

Table S5. Frequency of measurements for histograms.

\begin{tabular}{|c|c|c|c|c|c|c|c|c|c|c|c|c|c|c|c|c|c|c|c|c|c|c|c|c|c|c|c|c|c|c|c|c|c|c|c|c|c|c|c|c|c|}
\hline $\begin{array}{r}\text { DT Bin } \\
{[\mathrm{ms}]}\end{array}$ & 0 & 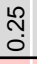 & مُ & $\begin{array}{l}\stackrel{L}{N} \\
0 \\
0\end{array}$ & -1 & $\stackrel{\text { N }}{\text { ָे }}$ & - & $\begin{array}{l}\stackrel{n}{\sim} \\
\stackrel{-}{-}\end{array}$ & $N$ & $\stackrel{\substack{N \\
\sim}}{\sim}$ & 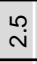 & $\begin{array}{l}\text { L } \\
\text { N } \\
\end{array}$ & $m$ & $\begin{array}{l}\stackrel{L}{N} \\
\stackrel{m}{n}\end{array}$ & ली & $\begin{array}{l}\frac{10}{N} \\
m \\
\end{array}$ & $\checkmark$ & $\begin{array}{l}\stackrel{\sim}{N} \\
\dot{v}\end{array}$ & $\stackrel{L}{+}$ & 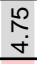 & L) & $\begin{array}{l}\stackrel{\sim}{N} \\
\stackrel{1}{n} \\
\end{array}$ & $\begin{array}{l}\text { L } \\
\text { L }\end{array}$ & $\begin{array}{l}\stackrel{2}{R} \\
10 \\
\end{array}$ & 0 & $\begin{array}{l}\stackrel{1}{0} \\
0 \\
0\end{array}$ & ए? & $\begin{array}{l}\stackrel{2}{R} \\
0 \\
\end{array}$ & 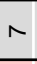 & $\stackrel{\text { N }}{N}$ & ᄂ? & $\stackrel{n}{\sim}$ & $\infty$ & 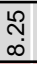 & $\begin{array}{l}\text { ? } \\
\infty\end{array}$ & $\begin{array}{l}\frac{1}{\Gamma} \\
\infty \\
\infty\end{array}$ & の) & $\begin{array}{l}\stackrel{2}{\sim} \\
\text { ஸे }\end{array}$ & $\stackrel{\text { ?̊ }}{\circ}$ & $\begin{array}{l}\frac{1}{\Gamma} \\
\sigma^{\circ}\end{array}$ & \\
\hline 3 & 0 & 0 & 0 & 0 & 0 & 0 & 0 & 0 & 0 & 0 & 0 & 2 & 7 & 14 & 31 & 41 & 58 & 42 & 15 & 3 & 0 & 0 & 0 & 0 & 0 & 0 & 0 & 0 & & 0 & 0 & 0 & & 0 & 0 & 0 & & 0 & 0 & 0 & \\
\hline bi & 0 & 0 & 0 & 0 & 0 & 0 & 0 & 0 & 1 & 3 & 18 & 42 & 66 & 61 & 17 & 2 & 0 & 0 & 0 & 0 & 0 & 0 & 0 & 0 & 0 & 0 & 0 & 0 & & 0 & & 0 & & 0 & & 0 & & 0 & 0 & 0 & \\
\hline & 0 & 0 & 0 & 0 & 0 & 0 & 0 & 0 & 0 & 0 & 0 & 0 & 0 & 0 & 0 & 0 & 0 & 0 & 0 & 0 & 0 & 0 & 1 & 1 & 3 & 7 & 4 & 11 & 18 & 33 & 25 & 27 & 39 & 22 & 17 & 2 & 0 & 0 & 0 & 0 & \\
\hline it & 0 & 0 & 0 & 0 & 0 & 0 & 0 & 0 & 0 & 0 & 0 & 0 & c & 0 & & 4 & & 15 & 41 & 46 & 54 & 33 & 7 & 0 & & & & & 0 & 0 & 0 & 0 & 0 & 0 & 0 & & 0 & 0 & 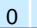 & 0 & \\
\hline
\end{tabular}




\section{(A)}

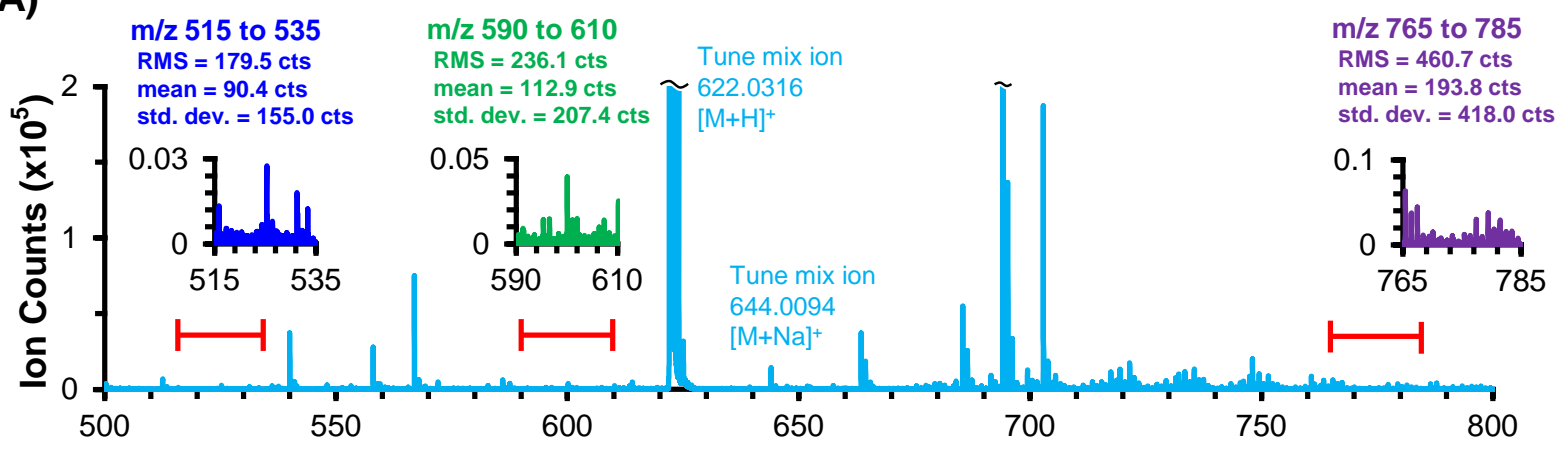

(B)

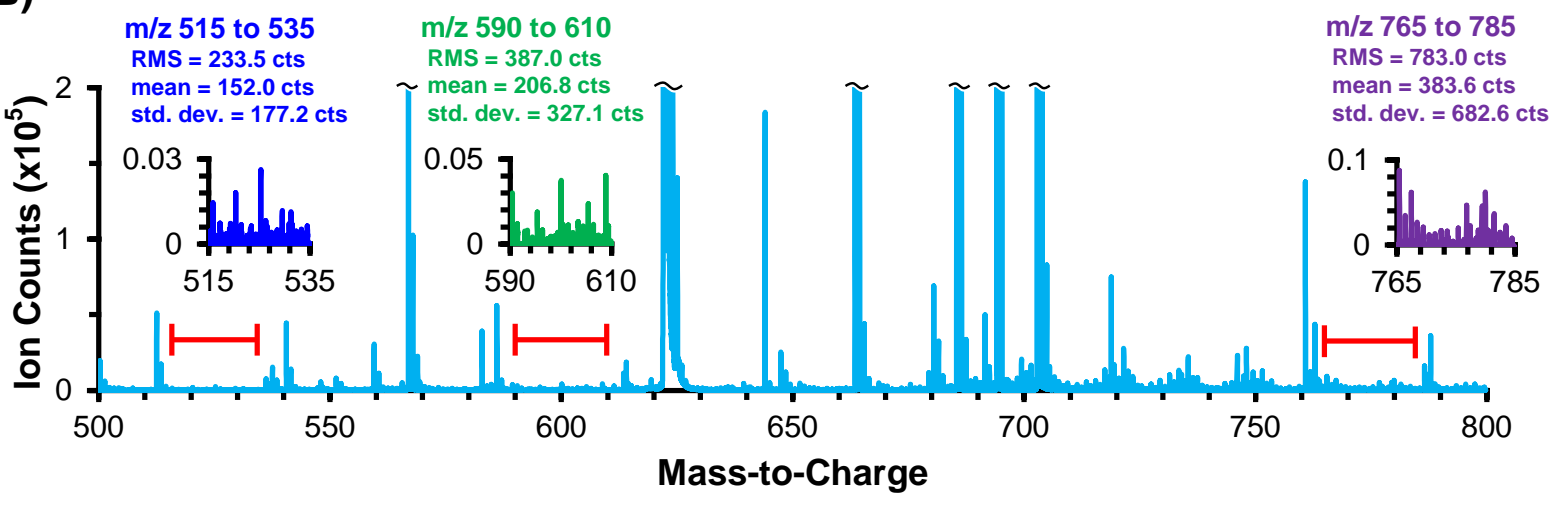

Figure S6. Estimation of baseline noise across three regions of the MS spectrum obtained in both (A) single pulse, and (B) 4-bit multiplexing mode. The insets highlight the ion signal contained within each corresponding $\mathrm{m} / \mathrm{z}$ region. 
Table S6. Summary of the spectral noise (in ion counts) obtained for each of three $\mathrm{m} / \mathrm{z}$ ranges for single pulse and multiplexed modes. The fold change in the root mean square (RMS) signal from each $\mathrm{m} / \mathrm{z}$ region for single pulse and 4-bit results is contained in the right column.

\begin{tabular}{|c|c|c|c|c|c|c|c|}
\hline \multirow[b]{2}{*}{$\mathrm{m} / \mathrm{z}$ range } & \multicolumn{3}{|c|}{ Single Pulse } & \multicolumn{3}{|c|}{ 4-bit Multiplexed } & \multirow{2}{*}{$\begin{array}{l}\text { Change in } \\
\text { RMS Counts } \\
\text { [fold change] }\end{array}$} \\
\hline & RMS & $\begin{array}{l}\text { Mean } \\
\text { [ion cour }\end{array}$ & Std. Dev. & RMS & $\begin{array}{l}\text { Mean } \\
\text { [ion cour }\end{array}$ & Std. Dev. & \\
\hline 515 to 535 & 179.5 & 90.4 & 155.0 & 233.5 & 152.0 & 177.2 & 1.3 \\
\hline 590 to 610 & 236.1 & 112.9 & 207.4 & 387.0 & 206.8 & 327.1 & 1.6 \\
\hline 765 to 785 & 460.7 & 193.8 & 418.0 & 783.0 & 383.6 & 682.6 & 1.7 \\
\hline AVERAGE & 292.1 & & & 467.8 & & & 1.5 \\
\hline
\end{tabular}


(A) Single Pulse Mode
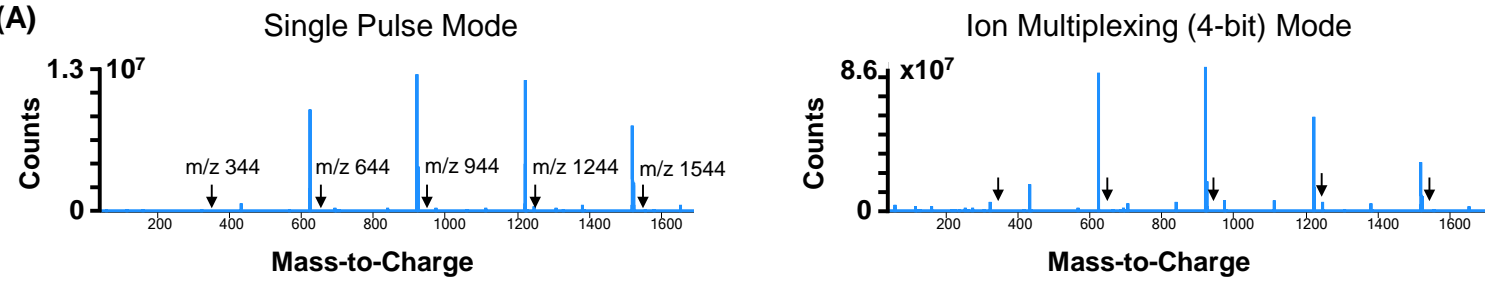

(B)

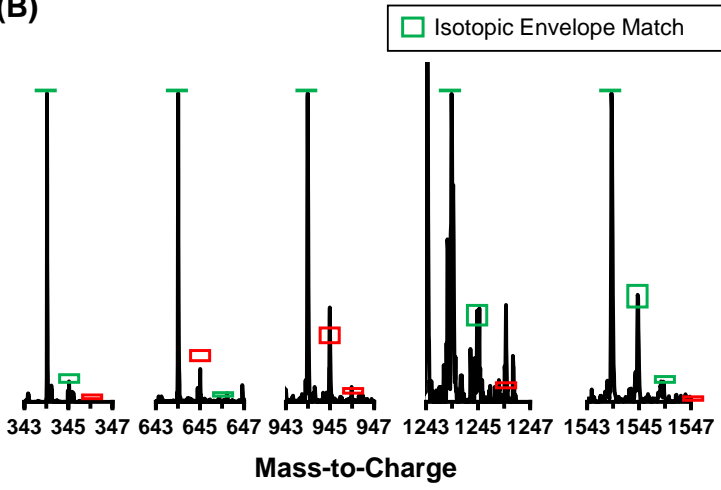

Isotopic Envelope Mismatch

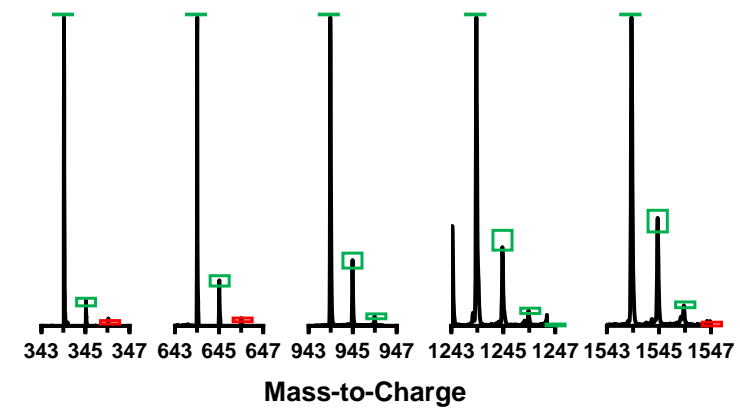

Figure S7. Example spectra illustrating the recovery of isotope information for 4-bit ion multiplexing operation. (A) Mass spectra for tune mix ions with the low-abundance sodium-adducted ion forms labeled. Results from both single pulse (left panel) and 4-bit multiplexing (right panel) acquired for the same acquisition time of 2 minutes are shown. (B) Expanded mass spectra for each of the sodium-coordinated tune mix ions obtained in each mode with the corresponding theoretical isotopic envelopes overlaid with the open boxes. Each box represents a $\pm 10 \%$ correspondence to the theoretical isotope ratio, with red boxes representing isotopes which do not match the theoreticallyexpected peak heights. Total ion counts for each spectral inset, from left to right, are as follows $\left(x 10^{3}\right): 8.7,2.8,1.1,0.9,2.7,61.8,19.3,8.1,6.8,19.7$. 
Table S7. Tabulated data for the recovery of isotope information for the sodium-coordinated tune mix ions. Text in green are relative peak heights which are within $\pm 10 \%$ of what is expected theoretically, while the text in red represent peak heights that do not correspond to the theoretical isotope ratios. Null (--) entries have theoretical abundances below $0.25 \%$ and thus are not observed with statistical significance in the MS analysis.

\begin{tabular}{|c|c|c|c|c|c|c|c|c|c|c|}
\hline \multirow{3}{*}{$\begin{array}{c}\text { Analyte } \\
\text { Ion }\end{array}$} & \multirow{2}{*}{$\begin{array}{l}\text { Exact } \\
\text { Mass }\end{array}$} & \multicolumn{3}{|c|}{ Theoretical } & \multicolumn{3}{|c|}{ Single Pulse } & \multicolumn{3}{|c|}{ 4-bit Multiplexed } \\
\hline & & $\stackrel{+}{+}$ & $\stackrel{\sim}{\stackrel{+}{\Sigma}}$ & $\stackrel{m}{\Sigma}$ & $\stackrel{-1}{+}$ & $\stackrel{\sim}{\stackrel{+}{\Sigma}}$ & $\stackrel{m}{\stackrel{m}{\Sigma}}$ & $\stackrel{-1}{+}$ & $\stackrel{\sim}{\stackrel{+}{\Sigma}}$ & $\stackrel{m}{\stackrel{m}{\Sigma}}$ \\
\hline & [Da] & \multicolumn{3}{|c|}{ [\% relative abundance] } & \multicolumn{3}{|c|}{$\begin{array}{c}{[\% \text { relative abundance }]} \\
\text { (\% match) }\end{array}$} & \multicolumn{3}{|c|}{$\begin{array}{c}\text { [\% relative abundance] } \\
\text { (\% match) }\end{array}$} \\
\hline $\mathrm{HP}-321+\mathrm{Na}$ & 344.0301 & 8.10 & 1.52 & 0.11 & $\begin{array}{l}7.83 \\
(3 \%) \\
\end{array}$ & $\begin{array}{l}0.85 \\
(44 \%) \\
\end{array}$ & -- & $\begin{array}{l}8.50 \\
(5 \%)\end{array}$ & $\begin{array}{l}2.02 \\
(33 \%)\end{array}$ & -- \\
\hline $\mathrm{HP}-621+\mathrm{Na}$ & 644.0109 & 14.59 & 2.22 & 0.22 & $\begin{array}{l}10.72 \\
(27 \%)\end{array}$ & $\begin{array}{l}2.02 \\
(9 \%)\end{array}$ & -- & $\begin{array}{c}14.77 \\
(1 \%)\end{array}$ & $\begin{array}{l}2.54 \\
(14 \%)\end{array}$ & -- \\
\hline $\mathrm{HP}-921+\mathrm{Na}$ & 943.9917 & 21.07 & 3.35 & 0.39 & $\begin{array}{l}30.60 \\
(45 \%) \\
\end{array}$ & $\begin{array}{l}4.85 \\
(45 \%) \\
\end{array}$ & $\begin{array}{c}0.97 \\
(149 \%)\end{array}$ & $\begin{array}{c}21.38 \\
(1 \%) \\
\end{array}$ & $\begin{array}{l}3.17 \\
(5 \%) \\
\end{array}$ & $\begin{array}{l}0.27 \\
(31 \%) \\
\end{array}$ \\
\hline $\mathrm{HP}-1221+\mathrm{Na}$ & 1243.9726 & 27.56 & 4.89 & 0.65 & $\begin{array}{c}29.59 \\
(7 \%) \\
\end{array}$ & $\begin{array}{l}7.12 \\
(46 \%) \\
\end{array}$ & $\begin{array}{l}1.12 \\
(73 \%) \\
\end{array}$ & $\begin{array}{c}25.50 \\
(7 \%) \\
\end{array}$ & $\begin{array}{l}5.35 \\
(9 \%) \\
\end{array}$ & $\begin{array}{l}0.64 \\
(2 \%) \\
\end{array}$ \\
\hline $\mathrm{HP}-1521+\mathrm{Na}$ & 1543.9534 & 34.05 & 6.85 & 1.02 & $\begin{array}{c}35.64 \\
(5 \%)\end{array}$ & $\begin{array}{l}6.68 \\
(2 \%)\end{array}$ & $\begin{array}{l}1.73 \\
(70 \%)\end{array}$ & $\begin{array}{c}35.00 \\
(3 \%)\end{array}$ & $\begin{array}{l}6.67 \\
(3 \%)\end{array}$ & $\begin{array}{l}1.86 \\
(83 \%)\end{array}$ \\
\hline
\end{tabular}




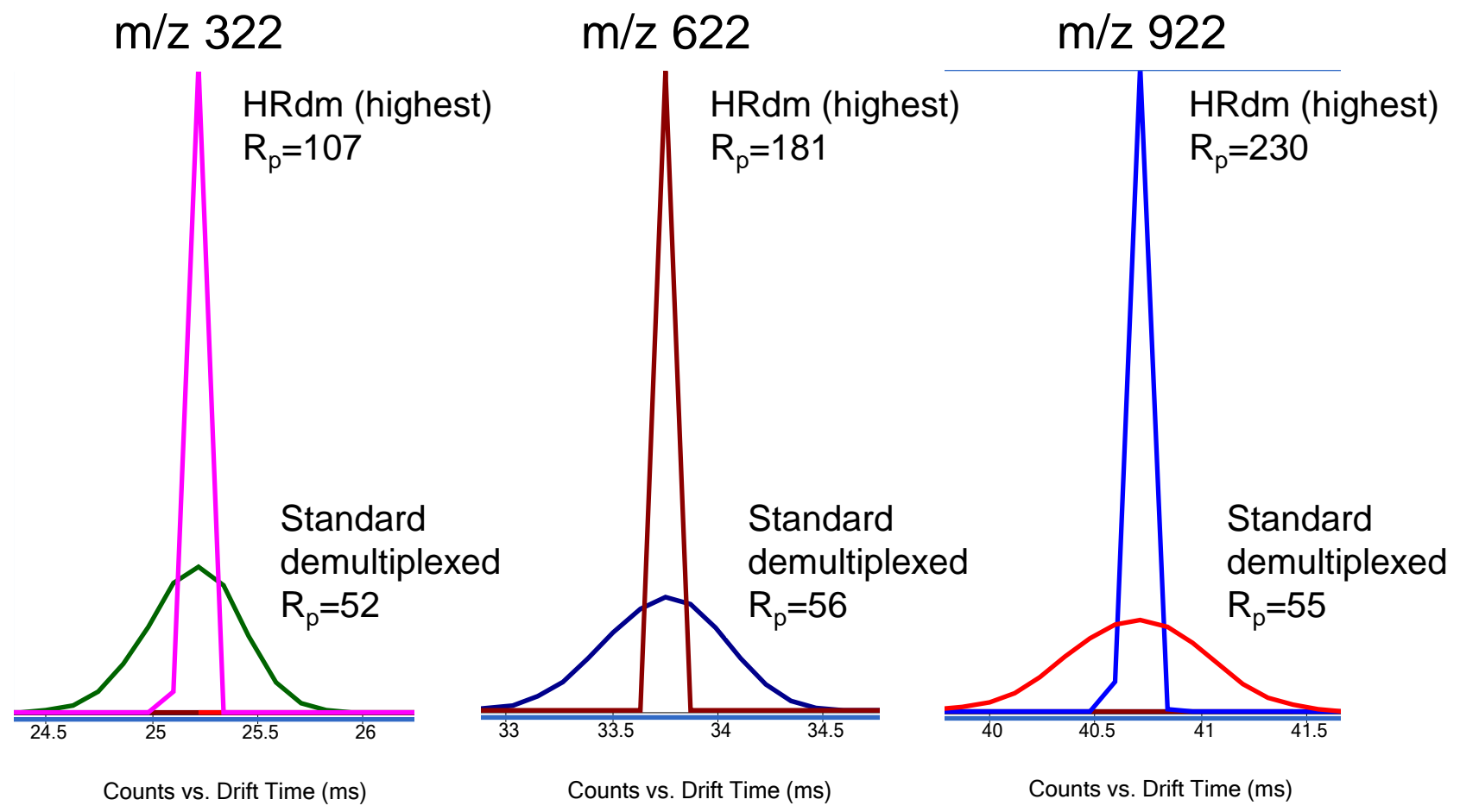

Figure S8. Overlaid IM peaks for three tune mix components obtained from both standard (4-bit) demultiplexing and HRdm (highest processing level). $R_{p}$ values are obtained from the vendor software. 

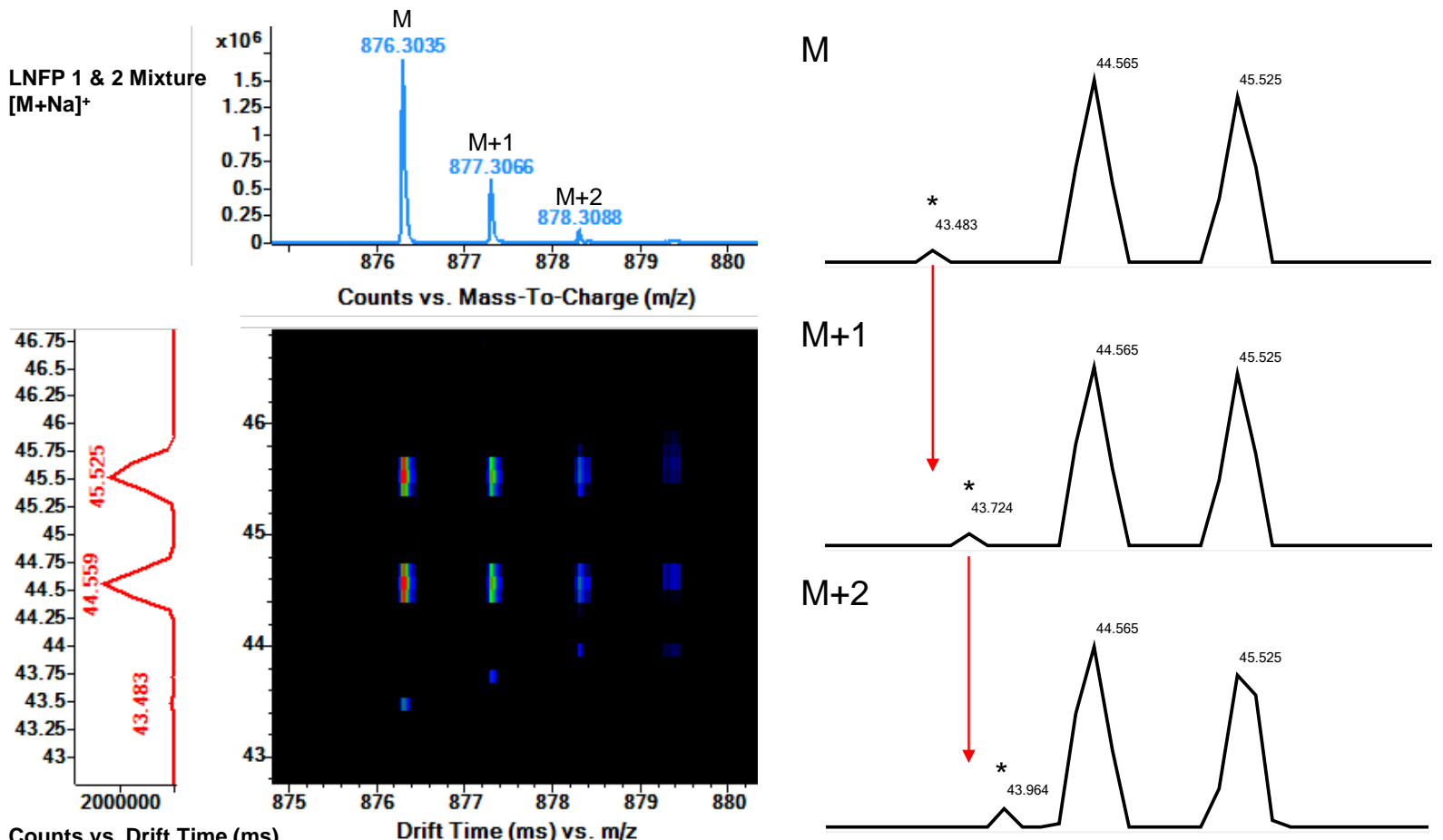

Figure S9. Example 2D IM-MS spectrum of a mixture of LNFP1 and LNFP2 obtained with 4-bit ion multiplexing and processed with HRdm (highest processing level, level 3). The extracted IM spectra for each of the three isotope peaks are shown in the right panel and illustrate a case where a prominent peak $\left({ }^{*}\right)$ appears in the spectra (here at low drift time), but does not correlate in arrival time across the different isotopes, which identifies this peak as a data artifact arising from HRdm. 
Table S8. CCS values for all ions investigated in this work.

\begin{tabular}{|c|c|c|c|c|c|c|c|c|c|c|}
\hline & \multirow{3}{*}{ Analyte } & \multirow{3}{*}{$\begin{array}{l}\text { Ion } \\
\text { Form }\end{array}$} & \multirow{3}{*}{$\mathbf{Z}$} & \multirow{3}{*}{$\begin{array}{c}\text { Exact lon } \\
\text { Mass-to- } \\
\text { Charge } \\
{[\mathrm{m} / \mathrm{z}]}\end{array}$} & & \multicolumn{5}{|c|}{ Collision Cross Section (CCS) Value } \\
\hline & & & & & & $\begin{array}{l}\text { Single } \\
\text { Pulse }\end{array}$ & $\begin{array}{l}\text { Standard } \\
\text { dm (4-bit) }\end{array}$ & $\begin{array}{c}\text { HRdm } \\
\text { Level } 2\end{array}$ & $\begin{array}{l}\text { HRdm } \\
\text { Level } 3\end{array}$ & $\begin{array}{l}\text { CCS Avg. } \\
\text { (SP \& dm) }\end{array}$ \\
\hline & & & & & & \multicolumn{5}{|c|}{ [square angstroms] } \\
\hline \multirow{24}{*}{ 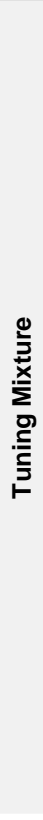 } & betaine & {$[\mathrm{M}+\mathrm{H}]^{+}$} & 1 & 118.0863 & $\operatorname{CCS}=$ & 122.82 & 122.22 & 121.30 & 121.30 & 122.52 \\
\hline & & & & & $\mathrm{SD}=$ & \pm 0.29 & \pm 0.17 & \pm 0.10 & \pm 0.20 & \pm 0.32 \\
\hline & & & & & $\% \mathrm{RSD}=$ & $0.23 \%$ & $0.14 \%$ & $0.08 \%$ & $0.16 \%$ & $0.26 \%$ \\
\hline & & & & & $\mathrm{N}=$ & 3 & 3 & 3 & 3 & 6 \\
\hline & HP-321 & {$[\mathrm{M}+\mathrm{H}]^{+}$} & 1 & 322.0481 & & 152.54 & 152.84 & 153.73 & 153.73 & 152.69 \\
\hline & & & & & & \pm 0.14 & \pm 0.14 & \pm 0.08 & \pm 0.07 & \pm 0.21 \\
\hline & & & & & & $0.09 \%$ & $0.09 \%$ & $0.05 \%$ & $0.05 \%$ & $0.14 \%$ \\
\hline & & & & & & 3 & 3 & 3 & 3 & 6 \\
\hline & HP-621 & {$[\mathrm{M}+\mathrm{H}]^{+}$} & 1 & 622.0290 & & 202.28 & 202.24 & 202.96 & 202.96 & 202.26 \\
\hline & & & & & & \pm 0.0 .07 & \pm 0.15 & \pm 0.12 & \pm 0.09 & \pm 0.26 \\
\hline & & & & & & $0.04 \%$ & $0.07 \%$ & $0.06 \%$ & $0.04 \%$ & $0.13 \%$ \\
\hline & & & & & & 3 & 3 & 3 & 3 & 6 \\
\hline & HP-921 & {$[\mathrm{M}+\mathrm{H}]^{+}$} & 1 & 922.0098 & & 243.22 & 243.20 & 243.64 & 243.64 & 243.21 \\
\hline & & & & & & \pm 0.11 & \pm 0.16 & \pm 0.10 & \pm 0.07 & \pm 0.28 \\
\hline & & & & & & $0.04 \%$ & $0.07 \%$ & $0.04 \%$ & $0.03 \%$ & $0.12 \%$ \\
\hline & & & & & & 3 & 3 & 3 & 3 & 6 \\
\hline & HP-1221 & {$[\mathrm{M}+\mathrm{H}]^{+}$} & 1 & 1221.9906 & & 282.29 & 282.30 & 282.20 & 282.20 & 282.29 \\
\hline & & & & & & \pm 0.16 & \pm 0.19 & \pm 0.20 & \pm 0.18 & \pm 0.32 \\
\hline & & & & & & $0.06 \%$ & $0.07 \%$ & $0.07 \%$ & $0.06 \%$ & $0.11 \%$ \\
\hline & & & & & & 3 & 3 & 3 & 3 & 6 \\
\hline & HP-1521 & {$[\mathrm{M}+\mathrm{H}]^{+}$} & 1 & 1521.9715 & & 317.59 & 317.54 & 316.96 & 316.96 & 317.57 \\
\hline & & & & & & \pm 0.16 & \pm 0.24 & \pm 0.23 & \pm 0.12 & \pm 0.59 \\
\hline & & & & & & $0.05 \%$ & $0.08 \%$ & $0.07 \%$ & $0.04 \%$ & $0.19 \%$ \\
\hline & & & & & & 3 & 3 & 3 & 3 & 6 \\
\hline \multirow{32}{*}{ 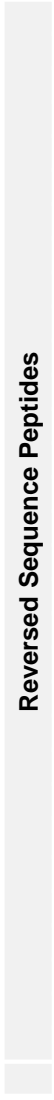 } & SDGRG & {$[\mathrm{M}+\mathrm{H}]^{+}$} & 1 & 491.1970 & & 203.46 & 203.66 & 203.62 & 203.50 & 203.54 \\
\hline & & & & & & \pm 0.23 & \pm 0.07 & \pm 0.04 & \pm 0.21 & \pm 0.24 \\
\hline & & & & & & $0.11 \%$ & $0.03 \%$ & $0.02 \%$ & $0.10 \%$ & $0.12 \%$ \\
\hline & & & & & & 3 & 3 & 3 & 3 & 6 \\
\hline & & {$[\mathrm{M}+\mathrm{Na}]^{+}$} & 1 & 513.1790 & & 202.35 & 202.49 & 202.48 & 202.50 & 202.40 \\
\hline & & & & & & \pm 0.12 & \pm 0.14 & \pm 0.22 & \pm 0.23 & \pm 0.30 \\
\hline & & & & & & $0.06 \%$ & $0.07 \%$ & $0.11 \%$ & $0.11 \%$ & $0.15 \%$ \\
\hline & & & & & & 3 & 3 & 3 & 3 & 6 \\
\hline & & {$[\mathrm{M}+\mathrm{K}]^{+}$} & 1 & 529.1529 & & 207.72 & 207.95 & 208.03 & 208.41 & 207.81 \\
\hline & & & & & & \pm 0.33 & \pm 0.22 & \pm 0.24 & \pm 0.41 & \pm 0.36 \\
\hline & & & & & & $0.16 \%$ & $0.11 \%$ & $0.12 \%$ & $0.20 \%$ & $0.17 \%$ \\
\hline & & & & & & 3 & 3 & 3 & 3 & 6 \\
\hline & & {$\left[\mathrm{M}-\mathrm{H}_{2} \mathrm{O}+\mathrm{H}\right]^{+}$} & 1 & 473.1865 & & 203.30 & 203.49 & 203.49 & 203.54 & 203.37 \\
\hline & & & & & & \pm 0.08 & \pm 0.32 & \pm 0.37 & \pm 0.12 & \pm 0.42 \\
\hline & & & & & & $0.04 \%$ & $0.16 \%$ & $0.18 \%$ & $0.06 \%$ & $0.21 \%$ \\
\hline & & & & & & 3 & 3 & 3 & 3 & 6 \\
\hline & & {$[\mathrm{M}+2 \mathrm{H}]^{+2}$} & 2 & 246.1022 & & 264.66 & 266.34 & 265.71 & 265.41 & 265.33 \\
\hline & & & & & & \pm 0.70 & \pm 0.50 & \pm 0.11 & \pm 0.54 & \pm 0.84 \\
\hline & & & & & & $0.26 \%$ & $0.19 \%$ & $0.04 \%$ & $0.20 \%$ & $0.32 \%$ \\
\hline & & & & & & 3 & 3 & 3 & 3 & 6 \\
\hline & & {$[\mathrm{M}+\mathrm{H}+\mathrm{Na}]^{+2}$} & 2 & 257.0931 & & 260.60 & 261.78 & 261.75 & 262.06 & 261.07 \\
\hline & & & & & & \pm 0.12 & \pm 0.12 & \pm 0.16 & \pm 0.30 & \pm 0.65 \\
\hline & & & & & & $0.05 \%$ & $0.05 \%$ & $0.06 \%$ & $0.12 \%$ & $0.25 \%$ \\
\hline & & & & & & 3 & 3 & 3 & 3 & 6 \\
\hline & & {$\left[\mathrm{M}-\mathrm{H}_{2} \mathrm{O}+2 \mathrm{H}\right]^{+2}$} & 2 & 237.0969 & & 254.95 & 256.26 & 255.97 & 255.83 & 255.47 \\
\hline & & & & & & \pm 0.24 & \pm 0.21 & \pm 0.24 & \pm 0.36 & \pm 0.74 \\
\hline & & & & & & $0.09 \%$ & $0.08 \%$ & $0.09 \%$ & $0.14 \%$ & $0.29 \%$ \\
\hline & & & & & & 3 & 3 & 3 & 3 & 6 \\
\hline & GRGDS & {$[\mathrm{M}+\mathrm{H}]^{+}$} & 1 & 491.1970 & & 205.28 & 205.58 & 205.58 & 205.59 & 205.43 \\
\hline & & & & & & \pm 0.25 & \pm 0.16 & \pm 0.21 & \pm 0.12 & \pm 0.23 \\
\hline & & & & & & $0.12 \%$ & $0.08 \%$ & $0.10 \%$ & $0.06 \%$ & $0.11 \%$ \\
\hline & & & & & & 3 & 3 & 3 & 3 & 6 \\
\hline
\end{tabular}


Table S8. Continued

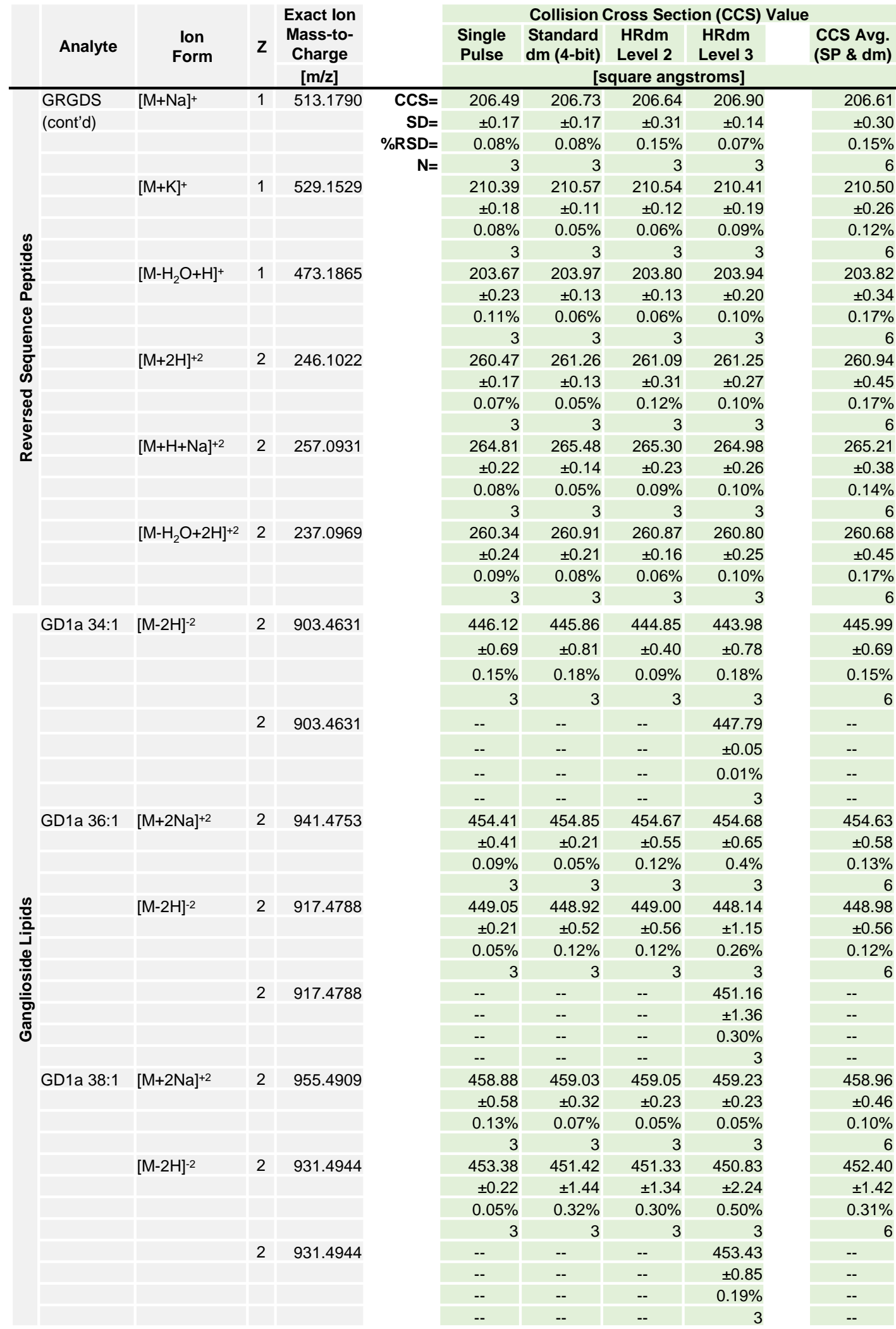


Table S8. Continued

\begin{tabular}{|c|c|c|c|c|c|c|c|c|c|c|}
\hline & \multirow{3}{*}{ Analyte } & \multirow{3}{*}{$\begin{array}{l}\text { lon } \\
\text { Form }\end{array}$} & \multirow{3}{*}{$\mathbf{z}$} & \multirow{3}{*}{$\begin{array}{c}\text { Exact lon } \\
\text { Mass-to- } \\
\text { Charge } \\
{[\mathrm{m} / \mathrm{z}]}\end{array}$} & & \multicolumn{5}{|c|}{ Collision Cross Section (CCS) Value } \\
\hline & & & & & & $\begin{array}{l}\text { Single } \\
\text { Pulse }\end{array}$ & $\begin{array}{l}\text { Standard } \\
\text { dm (4-bit) }\end{array}$ & $\begin{array}{l}\text { HRdm } \\
\text { Level } 2\end{array}$ & $\begin{array}{l}\text { HRdm } \\
\text { Level } 3\end{array}$ & $\begin{array}{l}\text { CCS Avg. } \\
\text { (SP \& dm) }\end{array}$ \\
\hline & & & & & & & & quare ang & troms] & \\
\hline \multirow{20}{*}{ 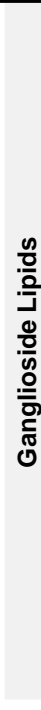 } & GD1b 34:1 & {$[\mathrm{M}-2 \mathrm{H}]^{-2}$} & 2 & 903.4631 & $\mathrm{CCS}=$ & 441.77 & 441.14 & 442.82 & 442.49 & 441.46 \\
\hline & & & & & $\mathrm{SD}=$ & \pm 0.62 & \pm 0.73 & \pm 0.36 & \pm 0.34 & \pm 0.70 \\
\hline & & & & & $\%$ RSD $=$ & $0.14 \%$ & $0.17 \%$ & $0.08 \%$ & $0.08 \%$ & $0.16 \%$ \\
\hline & & & & & $\mathrm{N}=$ & 3 & 3 & 3 & 3 & 6 \\
\hline & GD1b 36:1 & {$[\mathrm{M}+2 \mathrm{Na}]^{+2}$} & 2 & 941.4753 & & 471.74 & 471.66 & 471.54 & 471.75 & 471.70 \\
\hline & & & & & & \pm 0.40 & \pm 0.48 & \pm 0.27 & \pm 0.51 & \pm 0.46 \\
\hline & & & & & & $0.09 \%$ & $0.10 \%$ & $0.06 \%$ & $0.11 \%$ & $0.10 \%$ \\
\hline & & & & & & 3 & 3 & 3 & 3 & 6 \\
\hline & & {$[\mathrm{M}-2 \mathrm{H}]^{-2}$} & 2 & 917.4788 & & 446.31 & 446.28 & 446.48 & 445.92 & 446.30 \\
\hline & & & & & & \pm 0.63 & \pm 0.26 & \pm 0.32 & \pm 0.75 & \pm 0.43 \\
\hline & & & & & & $0.14 \%$ & $0.06 \%$ & $0.07 \%$ & $0.17 \%$ & $0.10 \%$ \\
\hline & & & & & & 3 & 3 & 3 & 3 & 6 \\
\hline & GD1b 38:1 & {$[\mathrm{M}+2 \mathrm{Na}]^{+2}$} & 2 & 955.4909 & & 476.57 & 476.28 & 475.69 & 476.00 & 476.43 \\
\hline & & & & & & \pm 0.40 & \pm 0.50 & \pm 0.40 & \pm 0.11 & \pm 0.51 \\
\hline & & & & & & $0.08 \%$ & $0.10 \%$ & $0.08 \%$ & $0.02 \%$ & $0.11 \%$ \\
\hline & & & & & & 3 & 3 & 3 & 3 & 6 \\
\hline & & {$[\mathrm{M}-2 \mathrm{H}]^{-2}$} & 2 & 931.4944 & & 450.57 & 450.38 & 450.61 & 449.85 & 450.38 \\
\hline & & & & & & \pm 0.36 & \pm 0.20 & \pm 0.25 & \pm 069 . & \pm 0.56 \\
\hline & & & & & & $0.08 \%$ & $0.04 \%$ & $0.06 \%$ & $0.15 \%$ & $0.12 \%$ \\
\hline & & & & & & 3 & 3 & 3 & 3 & 6 \\
\hline \multirow{36}{*}{ 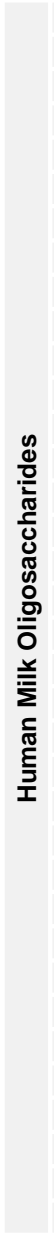 } & LNFP1 & {$[\mathrm{M}+\mathrm{Na}]^{+}$} & 1 & 876.2965 & & 274.76 & 274.90 & 274.83 & 274.96 & 274.83 \\
\hline & & & & & & \pm 0.26 & \pm 0.04 & \pm 0.10 & \pm 0.24 & \pm 0.38 \\
\hline & & & & & & $0.09 \%$ & $0.01 \%$ & $0.04 \%$ & $0.09 \%$ & $0.14 \%$ \\
\hline & & & & & & 3 & 3 & 3 & 3 & 6 \\
\hline & & {$[\mathrm{M}+\mathrm{K}]^{+}$} & 1 & 892.2695 & & -- & -- & -- & 273.65 & -- \\
\hline & & & & & & -- & -- & -- & \pm 0.39 & -- \\
\hline & & & & & & -- & -- & -- & $0.14 \%$ & -- \\
\hline & & & & & & -- & -- & -- & 3 & -- \\
\hline & & & 1 & 892.2695 & & 274.27 & 274.58 & 274.51 & 275.74 & 274.42 \\
\hline & & & & & & \pm 0.26 & \pm 0.10 & \pm 0.06 & \pm 0.35 & \pm 0.44 \\
\hline & & & & & & $0.09 \%$ & $0.04 \%$ & $0.02 \%$ & $0.13 \%$ & $0.16 \%$ \\
\hline & & & & & & 3 & 3 & 3 & 3 & 6 \\
\hline & & & 1 & 892.2695 & & 263.73 & 263.63 & 262.97 & 263.02 & 263.68 \\
\hline & & & & & & \pm 0.23 & \pm 0.18 & \pm 0.01 & \pm 0.07 & \pm 0.19 \\
\hline & & & & & & $0.09 \%$ & $0.07 \%$ & $0.01 \%$ & $0.03 \%$ & $0.07 \%$ \\
\hline & & & & & & 3 & 3 & 3 & 3 & 6 \\
\hline & & {$[\mathrm{M}+\mathrm{HK}]^{+2}$} & 2 & 446.6384 & & 311.13 & 312.45 & 312.60 & 312.75 & 311.79 \\
\hline & & & & & & \pm 0.15 & \pm 0.28 & \pm 0.36 & \pm 0.40 & \pm 0.75 \\
\hline & & & & & & $0.05 \%$ & $0.09 \%$ & $0.12 \%$ & $0.13 \%$ & $0.24 \%$ \\
\hline & & & & & & 3 & 3 & 3 & 3 & 6 \\
\hline & LNFP2 & {$[\mathrm{M}+\mathrm{Na}]^{+}$} & 1 & 876.2965 & & 268.79 & 269.17 & 268.91 & 269.05 & 268.98 \\
\hline & & & & & & \pm 0.24 & \pm 0.06 & \pm 0.16 & \pm 0.15 & \pm 0.26 \\
\hline & & & & & & $0.09 \%$ & $0.02 \%$ & $0.06 \%$ & $0.05 \%$ & $0.10 \%$ \\
\hline & & & & & & 3 & 3 & 3 & 3 & 6 \\
\hline & & {$[\mathrm{M}+\mathrm{K}]^{+}$} & 1 & 892.2695 & & 272.46 & 272.44 & 271.46 & 271.33 & 272.45 \\
\hline & & & & & & \pm 0.17 & \pm 0.28 & \pm 0.39 & \pm 0.37 & \pm 0.60 \\
\hline & & & & & & $0.06 \%$ & $0.10 \%$ & $0.14 \%$ & $0.14 \%$ & $0.22 \%$ \\
\hline & & & & & & 3 & 3 & 3 & 3 & 6 \\
\hline & & & & & & -- & -- & 275.82 & 275.86 & -- \\
\hline & & & & & & -- & -- & \pm 0.37 & \pm 0.40 & -- \\
\hline & & & & & & -- & -- & $0.14 \%$ & $0.15 \%$ & -- \\
\hline & & & & & & -- & -- & 3 & 3 & -- \\
\hline & & {$[\mathrm{M}+\mathrm{HK}]^{+2}$} & 2 & 446.6384 & & 308.19 & 309.58 & 309.02 & 308.40 & 308.89 \\
\hline & & & & & & \pm 0.37 & \pm 0.30 & \pm 0.41 & \pm 0.81 & \pm 0.82 \\
\hline & & & & & & $0.12 \%$ & $0.10 \%$ & $0.13 \%$ & $0.26 \%$ & $0.27 \%$ \\
\hline & & & & & & 3 & 3 & 3 & 3 & 6 \\
\hline
\end{tabular}


Table S8. Continued

\begin{tabular}{|c|c|c|c|c|c|c|c|c|c|c|}
\hline & \multirow{3}{*}{ Analyte } & \multirow{3}{*}{$\begin{array}{l}\text { Ion } \\
\text { Form }\end{array}$} & \multirow{3}{*}{$\mathbf{Z}$} & \multirow{3}{*}{$\begin{array}{c}\text { Exact lon } \\
\text { Mass-to- } \\
\text { Charge } \\
{[\mathrm{m} / \mathrm{z}]}\end{array}$} & & \multicolumn{5}{|c|}{ Collision Cross Section (CCS) Value } \\
\hline & & & & & & $\begin{array}{l}\text { Single } \\
\text { Pulse }\end{array}$ & $\begin{array}{l}\text { Standard } \\
\text { dm (4-bit) }\end{array}$ & $\begin{array}{l}\text { HRdm } \\
\text { Level } 2\end{array}$ & $\begin{array}{l}\text { HRdm } \\
\text { Level } 3\end{array}$ & $\begin{array}{l}\text { CCS Avg. } \\
\text { (SP \& dm) }\end{array}$ \\
\hline & & & & & & \multicolumn{5}{|c|}{ [square angstroms] } \\
\hline \multirow{16}{*}{ 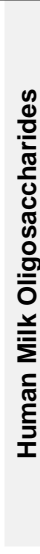 } & LNFP5 & {$[\mathrm{M}+\mathrm{Na}]^{+}$} & 1 & 876.2955 & $\mathrm{CCS}=$ & 271.76 & 272.30 & 272.19 & 272.31 & 272.03 \\
\hline & & & & & $\mathrm{SD}=$ & \pm 0.19 & \pm 0.07 & \pm 0.37 & \pm 0.21 & \pm 0.33 \\
\hline & & & & & $\%$ RSD $=$ & $0.07 \%$ & $0.03 \%$ & $0.13 \%$ & $0.08 \%$ & $0.12 \%$ \\
\hline & & & & & $\mathrm{N}=$ & 3 & 3 & 3 & 3 & 6 \\
\hline & & {$[\mathrm{M}+\mathrm{K}]^{+}$} & 1 & 892.2695 & & 273.72 & 274.36 & 276.12 & 276.03 & 274.04 \\
\hline & & & & & & \pm 0.12 & $\pm 0 . .05$ & \pm 0.19 & \pm 0.24 & \pm 0.36 \\
\hline & & & & & & $0.04 \%$ & $0.02 \%$ & $0.07 \%$ & $0.09 \%$ & $0.13 \%$ \\
\hline & & & & & & 3 & 3 & 3 & 3 & 6 \\
\hline & & & 1 & 892.2695 & & -- & -- & 272.09 & 271.92 & -- \\
\hline & & & & & & -- & -- & \pm 0.34 & \pm 0.29 & -- \\
\hline & & & & & & -- & -- & $0.12 \%$ & $0.11 \%$ & -- \\
\hline & & & & & & -- & -- & 3 & 3 & -- \\
\hline & & {$[\mathrm{M}+\mathrm{HK}]^{+2}$} & 2 & 446.6384 & & 305.36 & 306.81 & 306.47 & 306.40 & 306.09 \\
\hline & & & & & & \pm 0.17 & \pm 0.24 & \pm 0.70 & \pm 0.81 & \pm 0.82 \\
\hline & & & & & & $0.05 \%$ & $0.08 \%$ & $0.23 \%$ & $0.27 \%$ & $0.27 \%$ \\
\hline & & & & & & 3 & 3 & 3 & 3 & 6 \\
\hline \multirow{40}{*}{ 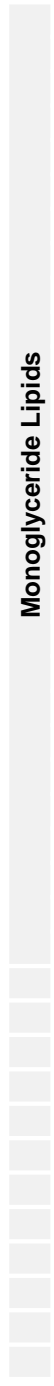 } & 1-LG & {$[\mathrm{M}+\mathrm{H}]^{+}$} & 1 & 355.2843 & & 196.42 & 196.37 & 196.54 & 196.54 & 196.39 \\
\hline & & & & & & \pm 0.24 & \pm 0.15 & \pm 0.14 & \pm 0.43 & \pm 0.28 \\
\hline & & & & & & $0.12 \%$ & $0.08 \%$ & $0.07 \%$ & $0.22 \%$ & $0.14 \%$ \\
\hline & & & & & & 3 & 3 & 3 & 3 & 6 \\
\hline & & {$[\mathrm{M}+\mathrm{Na}]^{+}$} & 1 & 377.2662 & & 192.20 & 192.34 & 192.35 & 191.77 & 192.27 \\
\hline & & & & & & \pm 0.25 & \pm 0.12 & \pm 0.11 & \pm 0.72 & \pm 0.31 \\
\hline & & & & & & $0.13 \%$ & $0.06 \%$ & $0.06 \%$ & $0.38 \%$ & $0.16 \%$ \\
\hline & & & & & & 3 & 3 & 3 & 3 & 6 \\
\hline & & {$[\mathrm{M}+\mathrm{K}]^{+}$} & 1 & 393.2402 & & 195.43 & 195.42 & 195.43 & 195.47 & 195.43 \\
\hline & & & & & & \pm 0.16 & \pm 0.13 & \pm 0.14 & \pm 0.23 & \pm 0.39 \\
\hline & & & & & & $0.08 \%$ & $0.06 \%$ & $0.07 \%$ & $0.12 \%$ & $0.20 \%$ \\
\hline & & & & & & 3 & 3 & 3 & 3 & 6 \\
\hline & 2-LG & {$[\mathrm{M}+\mathrm{H}]^{+}$} & 1 & 355.2843 & & 191.62 & 191.96 & 191.24 & 191.35 & 191.79 \\
\hline & & & & & & \pm 0.15 & \pm 0.10 & \pm 0.08 & \pm 0.35 & \pm 0.22 \\
\hline & & & & & & $0.08 \%$ & $0.05 \%$ & $0.04 \%$ & $0.19 \%$ & $0.12 \%$ \\
\hline & & & & & & 3 & 3 & 3 & 3 & 6 \\
\hline & & {$[\mathrm{M}+\mathrm{Na}]^{+}$} & 1 & 377.2662 & & 193.50 & 193.67 & 193.74 & 194.32 & 193.59 \\
\hline & & & & & & \pm 0.09 & \pm 0.12 & \pm 0.12 & \pm 0.40 & \pm 0.23 \\
\hline & & & & & & $0.05 \%$ & $0.06 \%$ & $0.06 \%$ & $0.21 \%$ & $0.12 \%$ \\
\hline & & & & & & 3 & 3 & 3 & 3 & 6 \\
\hline & & {$[\mathrm{M}+\mathrm{K}]^{+}$} & 1 & 393.2402 & & 197.47 & 197.50 & 197.54 & 197.89 & 197.49 \\
\hline & & & & & & \pm 0.06 & \pm 0.14 & \pm 0.13 & \pm 0.24 & \pm 0.30 \\
\hline & & & & & & $0.03 \%$ & $0.07 \%$ & $0.07 \%$ & $0.12 \%$ & $0.15 \%$ \\
\hline & & & & & & 3 & 3 & 3 & 3 & 6 \\
\hline & $1-A G$ & {$[\mathrm{M}+\mathrm{H}]^{+}$} & 1 & 379.2843 & & 197.47 & 197.61 & 198.40 & 197.97 & 197.54 \\
\hline & & & & & & \pm 0.14 & \pm 0.15 & \pm 0.54 & \pm 0.54 & \pm 0.23 \\
\hline & & & & & & $0.07 \%$ & $0.08 \%$ & $0.27 \%$ & $0.27 \%$ & $0.12 \%$ \\
\hline & & & & & & 3 & 3 & 3 & 3 & 6 \\
\hline & & {$[\mathrm{M}+\mathrm{Na}]^{+}$} & 1 & 401.2662 & & 196.47 & 196.31 & 196.33 & 196.52 & 196.39 \\
\hline & & & & & & \pm 0.22 & \pm 0.13 & \pm 0.12 & \pm 0.47 & \pm 0.22 \\
\hline & & & & & & $0.11 \%$ & $0.07 \%$ & $0.06 \%$ & $0.24 \%$ & $0.11 \%$ \\
\hline & & & & & & 3 & 3 & 3 & 3 & 6 \\
\hline & & {$[\mathrm{M}+\mathrm{K}]^{+}$} & 1 & 417.2401 & & 199.32 & 199.05 & 199.05 & 199.11 & 199.18 \\
\hline & & & & & & \pm 0.32 & \pm 0.13 & \pm 0.14 & \pm 0.16 & \pm 0.27 \\
\hline & & & & & & $0.16 \%$ & $0.07 \%$ & $0.07 \%$ & $0.08 \%$ & $0.14 \%$ \\
\hline & & & & & & 3 & 3 & 3 & 3 & 6 \\
\hline & 2-AG & {$[\mathrm{M}+\mathrm{H}]^{+}$} & 1 & 379.2843 & & 197.79 & 198.29 & 198.64 & 199.11 & 198.04 \\
\hline & & & & & & \pm 0.15 & \pm 0.13 & \pm 0.14 & \pm 0.15 & \pm 0.29 \\
\hline & & & & & & $0.08 \%$ & $0.07 \%$ & $0.07 \%$ & $0.08 \%$ & $0.14 \%$ \\
\hline & & & & & & 3 & 3 & 3 & 3 & 6 \\
\hline
\end{tabular}


Table S8. Continued

\begin{tabular}{|c|c|c|c|c|c|c|c|c|c|c|}
\hline & \multirow{3}{*}{ Analyte } & \multirow{3}{*}{$\begin{array}{l}\text { Ion } \\
\text { Form }\end{array}$} & \multirow{3}{*}{$\mathbf{Z}$} & \multirow{3}{*}{$\begin{array}{c}\text { Exact lon } \\
\text { Mass-to- } \\
\text { Charge } \\
{[\mathrm{m} / \mathrm{z}]}\end{array}$} & & \multicolumn{5}{|c|}{ Collision Cross Section (CCS) Value } \\
\hline & & & & & & $\begin{array}{l}\text { Single } \\
\text { Pulse }\end{array}$ & $\begin{array}{l}\text { Standard } \\
\text { dm (4-bit) }\end{array}$ & $\begin{array}{l}\text { HRdm } \\
\text { Level } 2\end{array}$ & $\begin{array}{l}\text { HRdm } \\
\text { Level } 3\end{array}$ & $\begin{array}{l}\text { CCS Avg. } \\
\text { (SP \& dm) }\end{array}$ \\
\hline & & & & & & \multicolumn{5}{|c|}{ [square angstroms] } \\
\hline \multirow{8}{*}{ 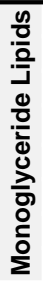 } & $2-A G$ & {$[\mathrm{M}+\mathrm{Na}]^{+}$} & 1 & 401.2662 & $\mathrm{CCS}=$ & 197.70 & 197.81 & 197.80 & 198.06 & 197.75 \\
\hline & - (cont'd) & & & & $\mathrm{SD}=$ & \pm 0.25 & \pm 0.13 & \pm 0.13 & \pm 0.32 & \pm 0.41 \\
\hline & & & & & \%RSD= & $0.13 \%$ & $0.07 \%$ & $0.07 \%$ & $0.16 \%$ & $0.21 \%$ \\
\hline & & & & & $\mathrm{N}=$ & 3 & 3 & 3 & 3 & 6 \\
\hline & & {$[\mathrm{M}+\mathrm{K}]^{+}$} & 1 & 417.2401 & & 200.92 & 201.25 & 201.20 & 201.52 & 201.09 \\
\hline & & & & & & \pm 0.17 & \pm 0.12 & \pm 0.14 & \pm 0.34 & \pm 0.40 \\
\hline & & & & & & $0.08 \%$ & $0.06 \%$ & $0.07 \%$ & $0.17 \%$ & $0.20 \%$ \\
\hline & & & & & & 3 & 3 & 3 & 3 & 6 \\
\hline \multirow{48}{*}{ 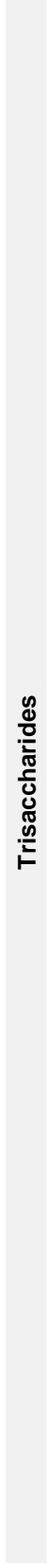 } & Melezitose & {$[\mathrm{M}+\mathrm{Na}]+$} & 1 & 527.1582 & & 201.94 & 201.77 & 201.78 & 201.80 & 201.85 \\
\hline & & & & & & \pm 0.04 & \pm 0.25 & \pm 0.38 & \pm 0.19 & \pm 0.39 \\
\hline & & & & & & $0.02 \%$ & $0.12 \%$ & $0.19 \%$ & $0.10 \%$ & $0.19 \%$ \\
\hline & & & & & & 3 & 3 & 3 & 3 & 6 \\
\hline & & {$[\mathrm{M}+\mathrm{K}]+$} & 1 & 543.1322 & & 202.44 & 202.22 & 202.35 & 202.31 & 202.33 \\
\hline & & & & & & \pm 0.05 & \pm 0.21 & \pm 0.39 & \pm 0.19 & \pm 0.38 \\
\hline & & & & & & $0.03 \%$ & $0.10 \%$ & $0.19 \%$ & $0.09 \%$ & $0.19 \%$ \\
\hline & & & & & & 3 & 3 & 3 & 3 & 6 \\
\hline & Kestose & {$[\mathrm{M}+\mathrm{Na}]+$} & 1 & 527.1582 & & 204.85 & 204.90 & 205.04 & 204.91 & 204.87 \\
\hline & & & & & & \pm 0.09 & \pm 0.03 & \pm 0.15 & \pm 0.09 & \pm 0.27 \\
\hline & & & & & & $0.04 \%$ & $0.01 \%$ & $0.07 \%$ & $0.04 \%$ & $0.13 \%$ \\
\hline & & & & & & 3 & 3 & 3 & 3 & 6 \\
\hline & & {$[\mathrm{M}+\mathrm{K}]+$} & 1 & 543.1322 & & 206.02 & 206.07 & 206.07 & 206.06 & 206.04 \\
\hline & & & & & & \pm 0.09 & \pm 0.02 & \pm 0.37 & \pm 0.37 & \pm 0.36 \\
\hline & & & & & & $0.04 \%$ & $0.01 \%$ & $0.18 \%$ & $0.18 \%$ & $0.17 \%$ \\
\hline & & & & & & 3 & 3 & 3 & 3 & 6 \\
\hline & Raffinose & {$[\mathrm{M}+\mathrm{Na}]+$} & 1 & 527.1582 & & 208.72 & 208.93 & 208.89 & 209.06 & 208.82 \\
\hline & & & & & & \pm 0.17 & \pm 0.17 & \pm 0.17 & \pm 0.21 & \pm 0.29 \\
\hline & & & & & & $0.08 \%$ & $0.08 \%$ & $0.08 \%$ & $0.10 \%$ & $0.14 \%$ \\
\hline & & & & & & 3 & 3 & 3 & 3 & 6 \\
\hline & & {$[\mathrm{M}+\mathrm{K}]+$} & 1 & 543.1322 & & 209.89 & 210.02 & 210.14 & 210.22 & 209.96 \\
\hline & & & & & & \pm 0.16 & \pm 0.17 & \pm 0.07 & \pm 0.24 & \pm 0.27 \\
\hline & & & & & & $0.08 \%$ & $0.08 \%$ & $0.04 \%$ & $0.11 \%$ & $0.13 \%$ \\
\hline & & & & & & 3 & 3 & 3 & 3 & 6 \\
\hline & Isomaltotriose & {$[\mathrm{M}+\mathrm{Na}]+$} & 1 & 527.1582 & & 210.73 & 210.94 & 210.94 & 210.99 & 210.83 \\
\hline & & & & & & \pm 0.16 & \pm 0.11 & \pm 0.18 & \pm 0.08 & \pm 0.35 \\
\hline & & & & & & $0.08 \%$ & $0.05 \%$ & $0.09 \%$ & $0.04 \%$ & $0.17 \%$ \\
\hline & & & & & & 3 & 3 & 3 & 3 & 6 \\
\hline & & {$[\mathrm{M}+\mathrm{K}]+$} & 1 & 543.1322 & & 211.86 & 211.92 & 211.81 & 211.89 & 211.89 \\
\hline & & & & & & \pm 0.13 & \pm 0.08 & \pm 0.42 & \pm 0.26 & \pm 0.39 \\
\hline & & & & & & $0.06 \%$ & $0.04 \%$ & $0.20 \%$ & $0.12 \%$ & $0.18 \%$ \\
\hline & & & & & & 3 & 3 & 3 & 3 & 6 \\
\hline & Maltotriose & {$[\mathrm{M}+\mathrm{Na}]+$} & 1 & 527.1582 & & 212.80 & 213.02 & 213.10 & 213.06 & 212.91 \\
\hline & & & & & & \pm 0.19 & \pm 0.05 & \pm 0.15 & \pm 0.11 & \pm 0.37 \\
\hline & & & & & & $0.09 \%$ & $0.03 \%$ & $0.07 \%$ & $0.05 \%$ & $0.17 \%$ \\
\hline & & & & & & 3 & 3 & 3 & 3 & 6 \\
\hline & & {$[\mathrm{M}+\mathrm{K}]+$} & 1 & 543.1322 & & -- & -- & -- & 212.93 & -- \\
\hline & & & & & & -- & -- & -- & \pm 0.09 & -- \\
\hline & & & & & & -- & -- & -- & $0.04 \%$ & -- \\
\hline & & & & & & -- & -- & -- & 3 & -- \\
\hline & & & 1 & 543.1322 & & 214.41 & 214.50 & 214.70 & 215.29 & 214.46 \\
\hline & & & & & & \pm 0.11 & \pm 0.09 & \pm 0.16 & \pm 0.11 & \pm 0.39 \\
\hline & & & & & & $0.05 \%$ & $0.04 \%$ & $0.07 \%$ & $0.05 \%$ & $0.18 \%$ \\
\hline & & & & & & 3 & 3 & 3 & 3 & 6 \\
\hline & Cellotriose & {$[\mathrm{M}+\mathrm{Na}]+$} & 1 & 527.1582 & & 216.01 & 216.12 & 216.13 & 216.03 & 216.07 \\
\hline & & & & & & \pm 0.06 & \pm 0.13 & \pm 0.25 & \pm 0.10 & \pm 0.31 \\
\hline & & & & & & $0.03 \%$ & $0.06 \%$ & $0.12 \%$ & $0.05 \%$ & $0.14 \%$ \\
\hline & & & & & & 3 & 3 & 3 & 3 & 6 \\
\hline
\end{tabular}


Table S8. Continued

\begin{tabular}{|c|c|c|c|c|c|c|c|c|c|c|}
\hline & \multirow{3}{*}{ Analyte } & \multirow{3}{*}{$\begin{array}{l}\text { Ion } \\
\text { Form }\end{array}$} & \multirow{3}{*}{$\mathbf{Z}$} & \multirow{3}{*}{$\begin{array}{c}\text { Exact Ion } \\
\text { Mass-to- } \\
\text { Charge } \\
{[\mathrm{m} / \mathrm{z}]}\end{array}$} & & \multicolumn{5}{|c|}{ Collision Cross Section (CCS) Value } \\
\hline & & & & & & $\begin{array}{l}\text { Single } \\
\text { Pulse }\end{array}$ & $\begin{array}{l}\text { Standard } \\
\text { dm (4-bit) }\end{array}$ & $\begin{array}{l}\text { HRdm } \\
\text { Level } 2\end{array}$ & $\begin{array}{l}\text { HRdm } \\
\text { Level } 3\end{array}$ & $\begin{array}{l}\text { CCS Avg. } \\
\text { (SP \& dm) }\end{array}$ \\
\hline & & & & & & \multicolumn{5}{|c|}{ [square angstroms] } \\
\hline \multirow{20}{*}{ 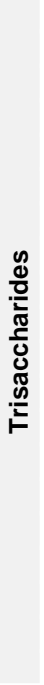 } & Cellotriose & {$[\mathrm{M}+\mathrm{K}]+$} & 1 & 543.1322 & $\mathrm{CCS}=$ & 216.78 & 216.89 & 216.70 & 216.62 & 216.83 \\
\hline & (cont'd) & & & & SD= & \pm 0.12 & \pm 0.15 & \pm 0.32 & \pm 0.37 & \pm 0.34 \\
\hline & & & & & \%RSD= & $0.05 \%$ & $0.07 \%$ & $0.15 \%$ & $0.17 \%$ & $0.16 \%$ \\
\hline & & & & & $N=$ & 3 & 3 & 3 & 3 & 6 \\
\hline & Panose & {$[\mathrm{M}+\mathrm{Na}]+$} & 1 & 527.1582 & & 208.89 & 208.93 & 208.89 & 208.05 & 208.91 \\
\hline & & & & & & \pm 0.09 & \pm 0.12 & \pm 0.07 & \pm 0.08 & \pm 0.30 \\
\hline & & & & & & $0.05 \%$ & $0.06 \%$ & $0.03 \%$ & $0.04 \%$ & $0.14 \%$ \\
\hline & & & & & & 3 & 3 & 3 & 3 & 6 \\
\hline & & & 1 & 527.1582 & & -- & -- & -- & 209.51 & -- \\
\hline & & & & & & -- & -- & -- & \pm 0.13 & -- \\
\hline & & & & & & -- & -- & -- & $0.06 \%$ & -- \\
\hline & & & & & & -- & -- & -- & 3 & -- \\
\hline & & {$[\mathrm{M}+\mathrm{K}]+$} & 1 & 543.1322 & & 205.61 & 205.87 & 205.69 & 205.65 & 205.74 \\
\hline & & & & & & \pm 0.20 & \pm 0.22 & \pm 0.20 & \pm 0.12 & \pm 0.44 \\
\hline & & & & & & $0.10 \%$ & $0.11 \%$ & $0.10 \%$ & $0.06 \%$ & $0.21 \%$ \\
\hline & & & & & & 3 & 3 & 3 & 3 & 6 \\
\hline & & & 1 & 543.1322 & & 210.41 & 210.61 & 211.05 & 210.83 & 210.51 \\
\hline & & & & & & \pm 0.23 & \pm 0.17 & \pm 0.39 & \pm 0.11 & \pm 0.31 \\
\hline & & & & & & $0.11 \%$ & $0.08 \%$ & $0.18 \%$ & $0.05 \%$ & $0.15 \%$ \\
\hline & & & & & & 3 & 3 & 3 & 3 & 6 \\
\hline
\end{tabular}

${ }^{*}$ CCS values are obtained from a single-field calibration relationship, ${ }^{6}$ and are averaged across triplicate, intra-day measurements at a drift field of $13.4 \mathrm{~V} / \mathrm{cm}$. The CCS Average (last column) is averaged across the single pulse and standard demultiplexed (dm) measurements ( $\mathrm{N}=6$ replicates), as $\mathrm{HRdm}$ often resolves additional features that do not align with the lower resolution results. LNFP5 and Panose measurements are summarized here, but were not included in the original study as these analytes exhibit complex distributions in the IM analysis. 
Human Milk Oligosaccharides - Lacto-N-Fucopentaose

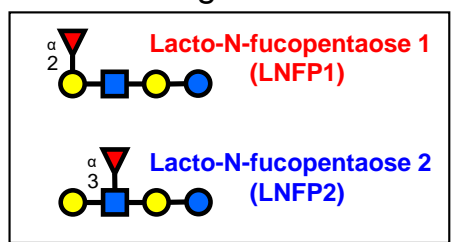

\begin{tabular}{|c|c|c|c|c|c|}
\hline \multicolumn{2}{|r|}{ Analyte } & Isomerism & $\begin{array}{l}\text { Molecular } \\
\text { Formula }\end{array}$ & $\begin{array}{l}\text { lon } \\
\text { Forms }\end{array}$ & $\begin{array}{c}\text { Exact } m / z \\
\text { Value }\end{array}$ \\
\hline LNFP1 & $\begin{array}{l}\text { Fuc( }(\alpha 1,2)-G a l(\beta 1,3)- \\
\operatorname{GIcNAc}(\beta 1,3)-G a l(\beta 1,4)-G I c\end{array}$ & \multirow{2}{*}{$\begin{array}{l}\text { mono- } \\
\text { saccharide } \\
\text { position and } \\
\text { linkage }\end{array}$} & \multirow{2}{*}{$\mathrm{C}_{32} \mathrm{H}_{55} \mathrm{NO}_{25}$} & \multirow{2}{*}{$\begin{array}{l}{[\mathrm{M}+\mathrm{Na}]^{+}} \\
{[\mathrm{M}+\mathrm{K}]^{+}}\end{array}$} & \multirow{2}{*}{$\begin{array}{l}876.2955 \\
892.2695\end{array}$} \\
\hline LNFP2 & $\begin{array}{l}\text { Gal-( }(\beta 1,3)-[\operatorname{Fuc}(\alpha 1,4)]- \\
\text { GIcNAc( }(\beta 1,3)-G a(\beta 1,4)-G l c\end{array}$ & & & & \\
\hline
\end{tabular}

$[\mathrm{M}+\mathrm{Na}]^{+}, \mathrm{m} / \mathrm{z} 876.2955$

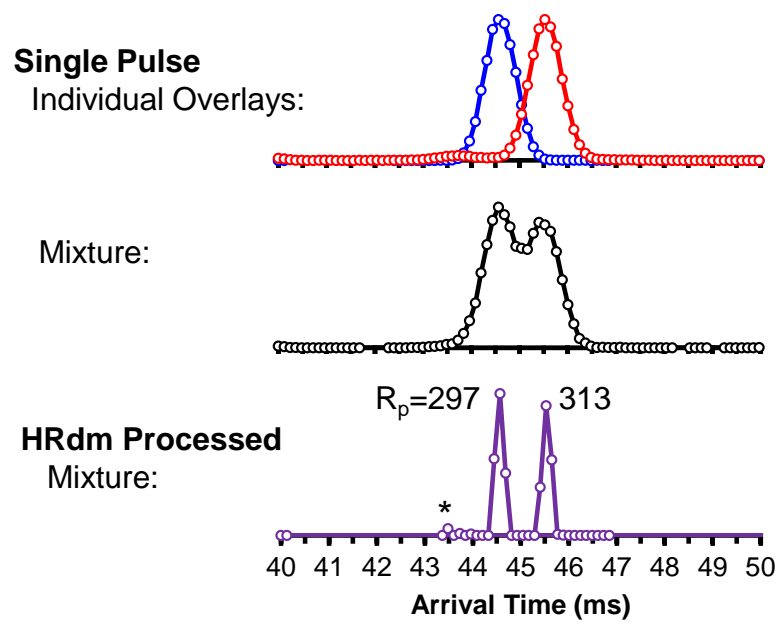

$[\mathrm{M}+\mathrm{K}]^{+}, \mathrm{m} / \mathrm{z} 892.2695$

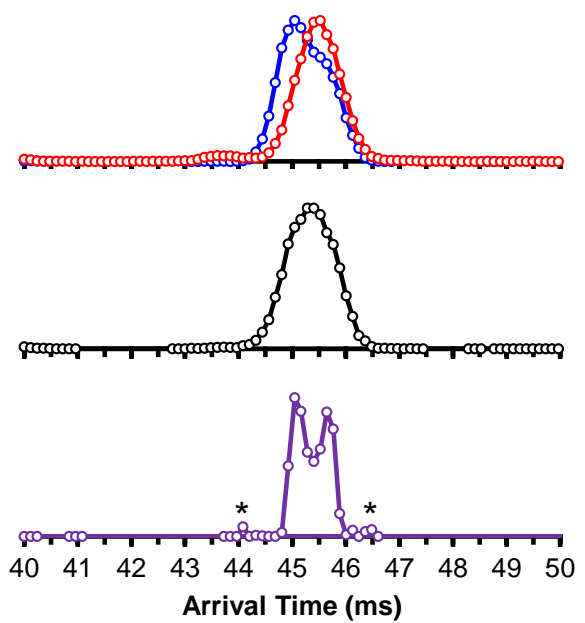

Figure S10. IM spectra (single pulse and HRdm) for the human milk oligosaccharides, LNFP1 and LNFP2. Here and elsewhere, the individual overlays are normalized to one another and the asterisks $\left.{ }^{*}\right)$ denote post-processing spectral artifacts. The resolving power $\left(R_{p}\right)$ annotated in the HRdm processed results (highest, level 3 ) were calculated from the software and, due to the limited peak sampling, do not necessarily reflect the true resolving power of the spectrum. 


\begin{tabular}{|c|c|c|c|c|c|}
\hline & & & & & \\
\hline \multirow{3}{*}{ Disialoganglioside GD1a (d18:1/16:0)} & Analyte & Isomerism & $\begin{array}{l}\text { Molecular } \\
\text { Formula }\end{array}$ & $\begin{array}{l}\text { Ion Forms } \\
\text { Observed }\end{array}$ & $\begin{array}{c}\text { Exact } m / z \\
\text { Values }\end{array}$ \\
\hline & GD1a (34:1) & \multirow{2}{*}{$\begin{array}{l}\text { sialic acid } \\
\text { position on } \\
\text { glycosylation }\end{array}$} & \multirow{2}{*}{$\mathrm{C}_{82} \mathrm{H}_{144} \mathrm{~N}_{4} \mathrm{O}_{39}$} & \multirow{2}{*}[\mathrm{M}-2\mathrm{H}]{$^{-2}$} & \multirow{2}{*}{903.4631} \\
\hline & GD1b (34:1) & & & & \\
\hline
\end{tabular}

$[\mathrm{M}-2 \mathrm{H}]^{-2}, \mathrm{~m} / \mathrm{z} 903.4631$

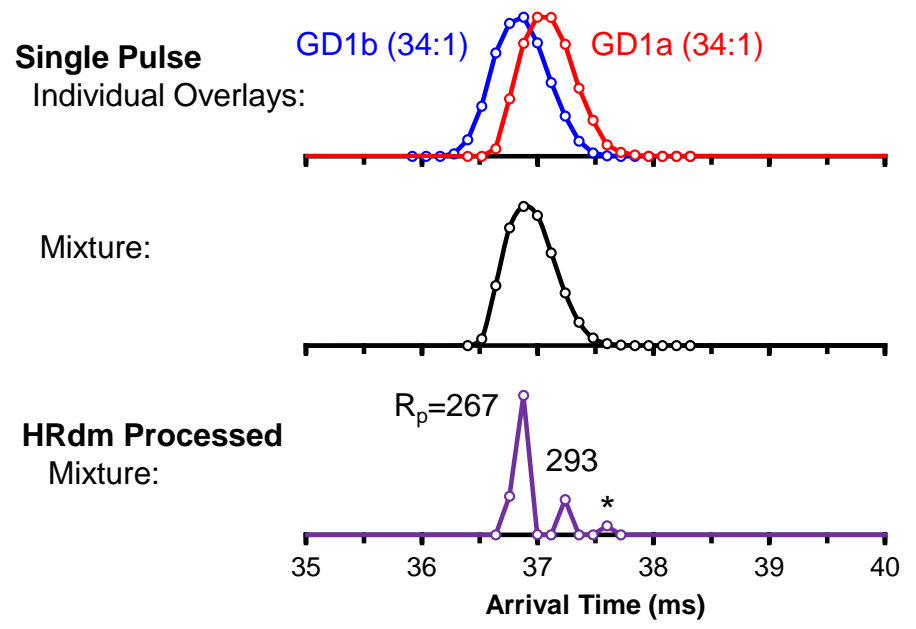

Figure S11. IM spectra (single pulse and HRdm) for the 34-carbon gangliosides, GD1a 34:1 and GD1b 34:1. Here, only the double-deprotonated ion is shown, as the doubly-sodiated ion form was not present in high enough abundance to provide good quality IM results. 


Gangliosides (36:1)
(3)

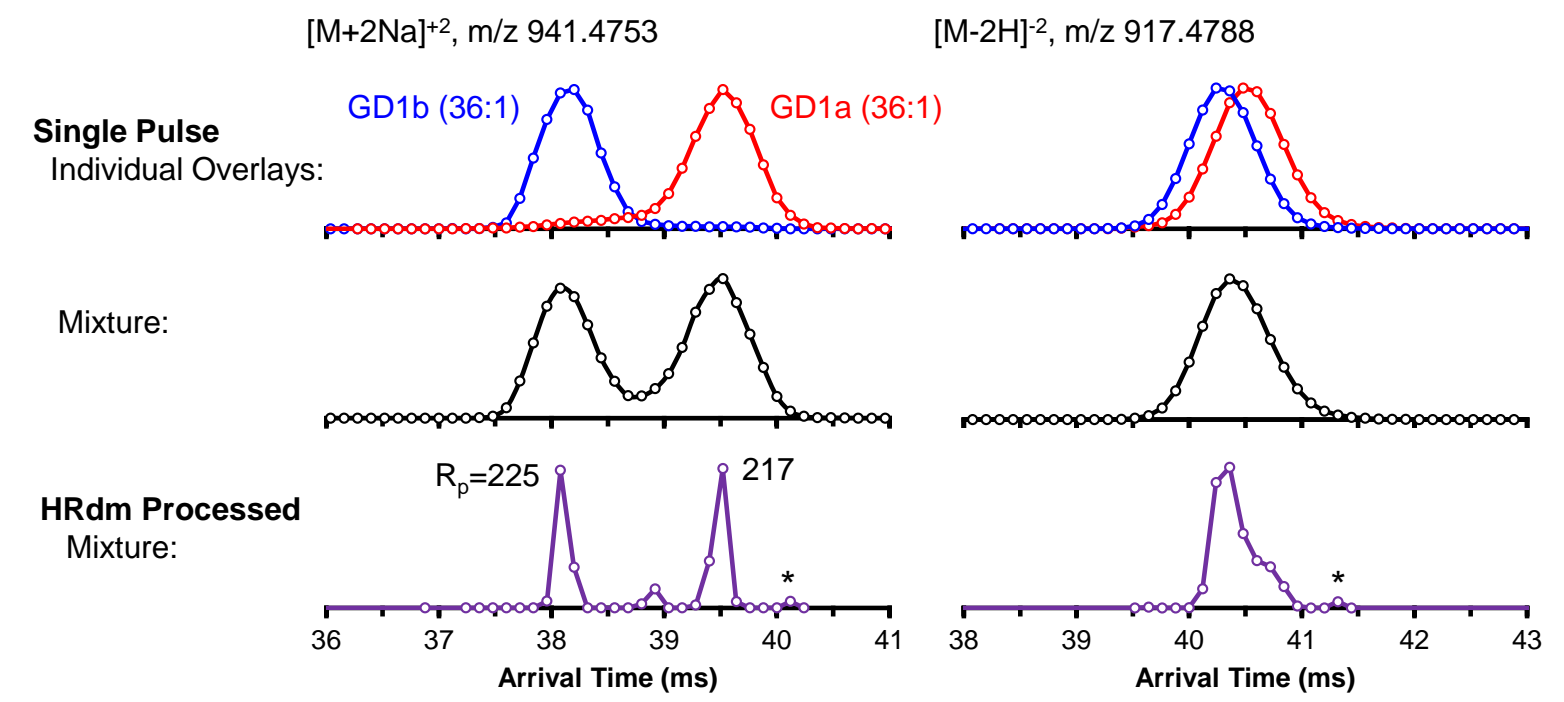

Figure S12. IM spectra (single pulse and HRdm) for the 36-carbon gangliosides GD1a 36:1 and GD1b $36: 1$. 


\begin{tabular}{l} 
Gangliosides (38:1) \\
\cline { 2 - 6 }
\end{tabular}

$[\mathrm{M}+2 \mathrm{Na}]^{+2}, \mathrm{~m} / \mathrm{z} 955.4909$

$[\mathrm{M}-2 \mathrm{H}]^{-2}, \mathrm{~m} / \mathrm{z} 931.4944$
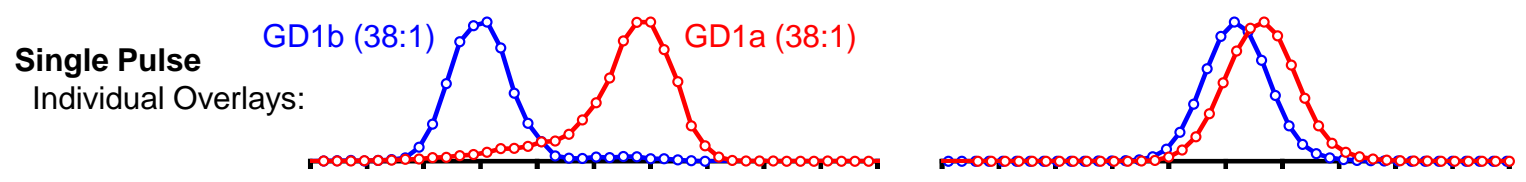

Mixture:
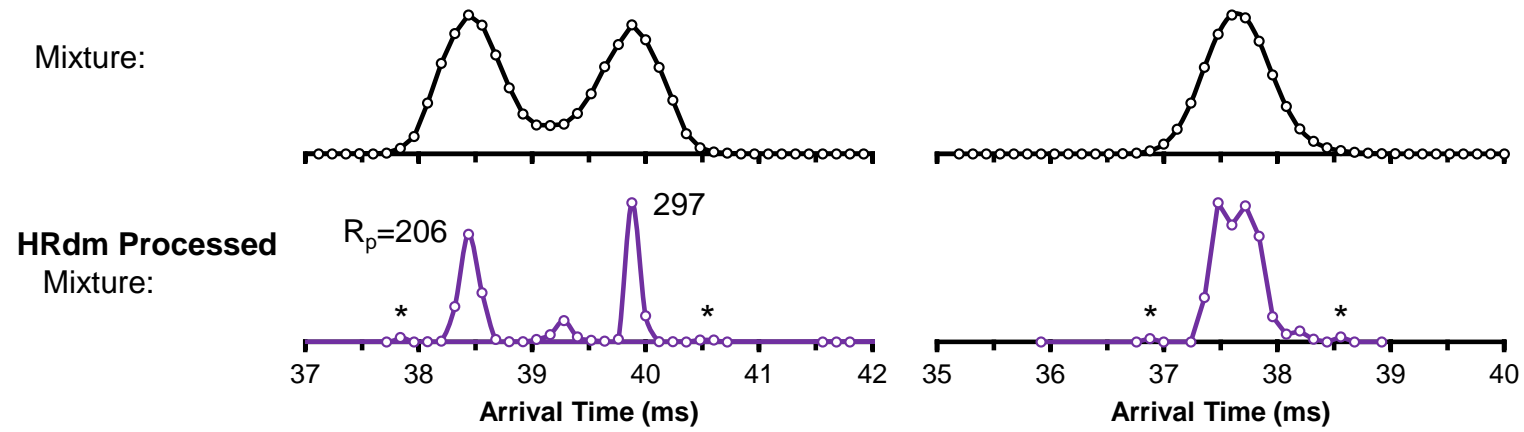

Figure S13. IM spectra (single pulse and HRdm) for the 38-carbon gangliosides, GD1a 38:1 and GD1b $38: 1$. 
Monoglyceride - Arachidonoyl Glycerol (20:4)

\begin{tabular}{|c|c|c|c|c|c|}
\hline 1-Arachidonoyl Glycero & Analyte & Isomerism & $\begin{array}{c}\text { Molecular } \\
\text { Formula }\end{array}$ & $\begin{array}{l}\text { Ion } \\
\text { Form }\end{array}$ & $\begin{array}{c}\text { Exact } \mathrm{m} / \mathrm{z} \\
\text { Value }\end{array}$ \\
\hline $\mathrm{N}=$ & $1-A G(20: 4)$ & \multirow{2}{*}{$\begin{array}{l}\text { sn- position of } \\
\text { the acyl tail }\end{array}$} & \multirow{2}{*}{$\mathrm{C}_{23} \mathrm{H}_{38} \mathrm{O}_{4}$} & \multirow{2}{*}[\mathrm{M}+\mathrm{Na}]{$^{+}$} & \multirow{2}{*}{401.2662} \\
\hline $\begin{array}{l}\text { 2-Arachidonoyl Glycerol (2-AG) Ho } \\
(20: 4)\end{array}$ & $2-A G(20: 4)$ & & & & \\
\hline
\end{tabular}

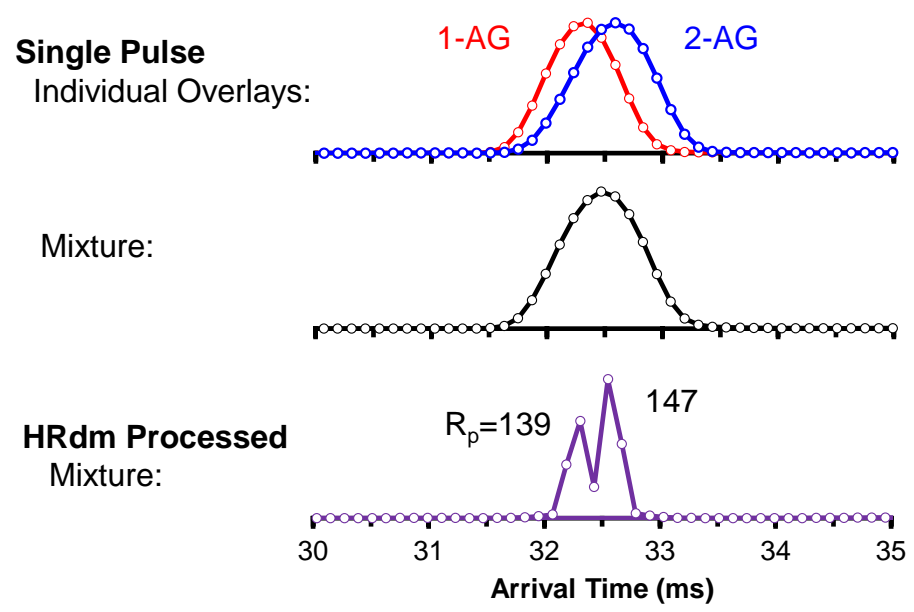

Figure S14. IM spectra (single pulse and HRdm) for the 20-carbon monoglycerides, 1-AG and 2-AG. 
Trisaccharides

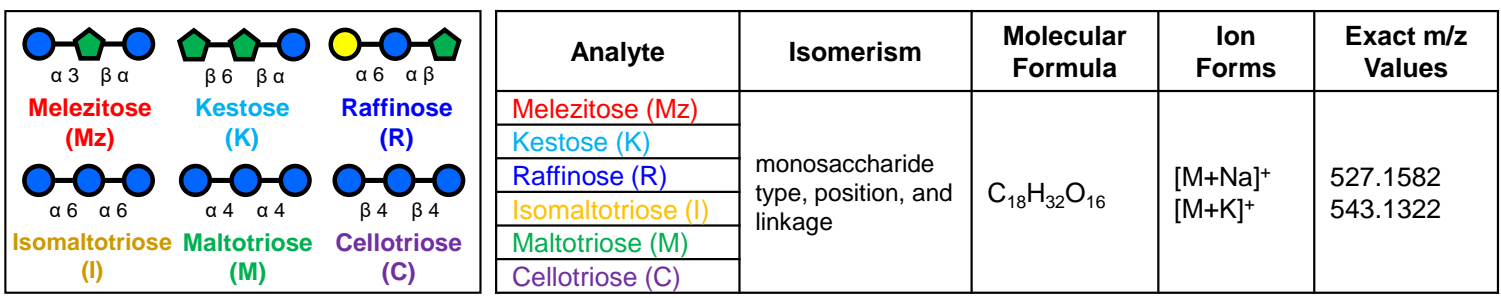

(A)

$[\mathrm{M}+\mathrm{Na}]^{+}, \mathrm{m} / \mathrm{z} 527.1582$
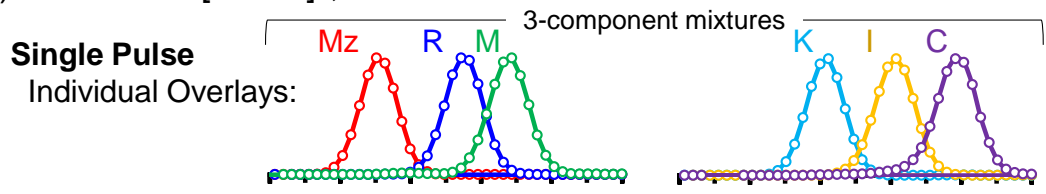

Mixture:
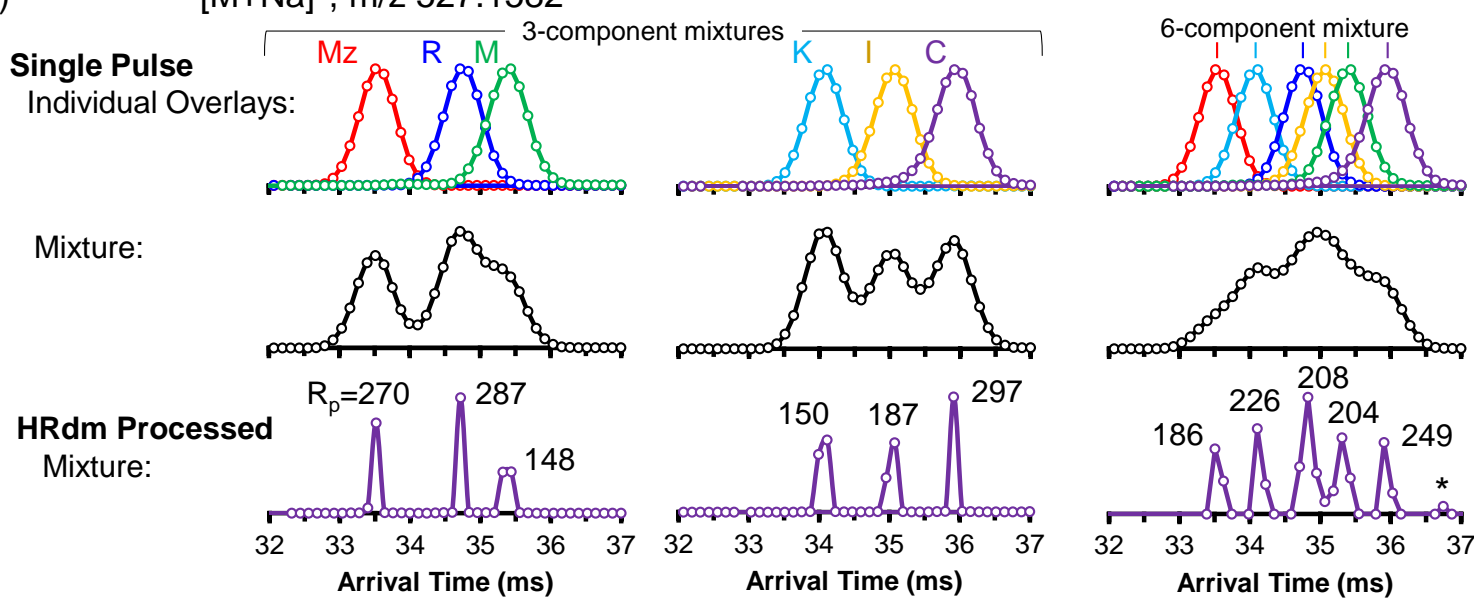

Trisaccharides

(B)

$[\mathrm{M}+\mathrm{K}]^{+}, \mathrm{m} / \mathrm{z} 543.1322$
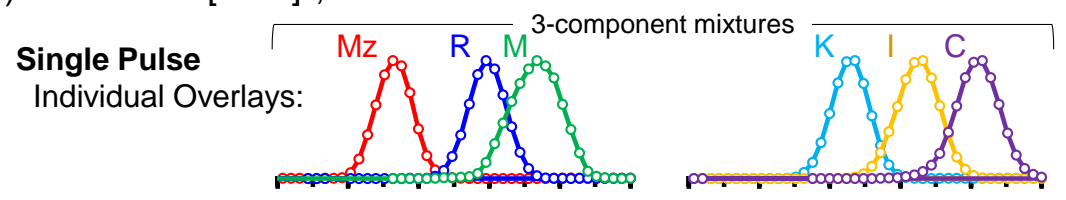

Mixture:
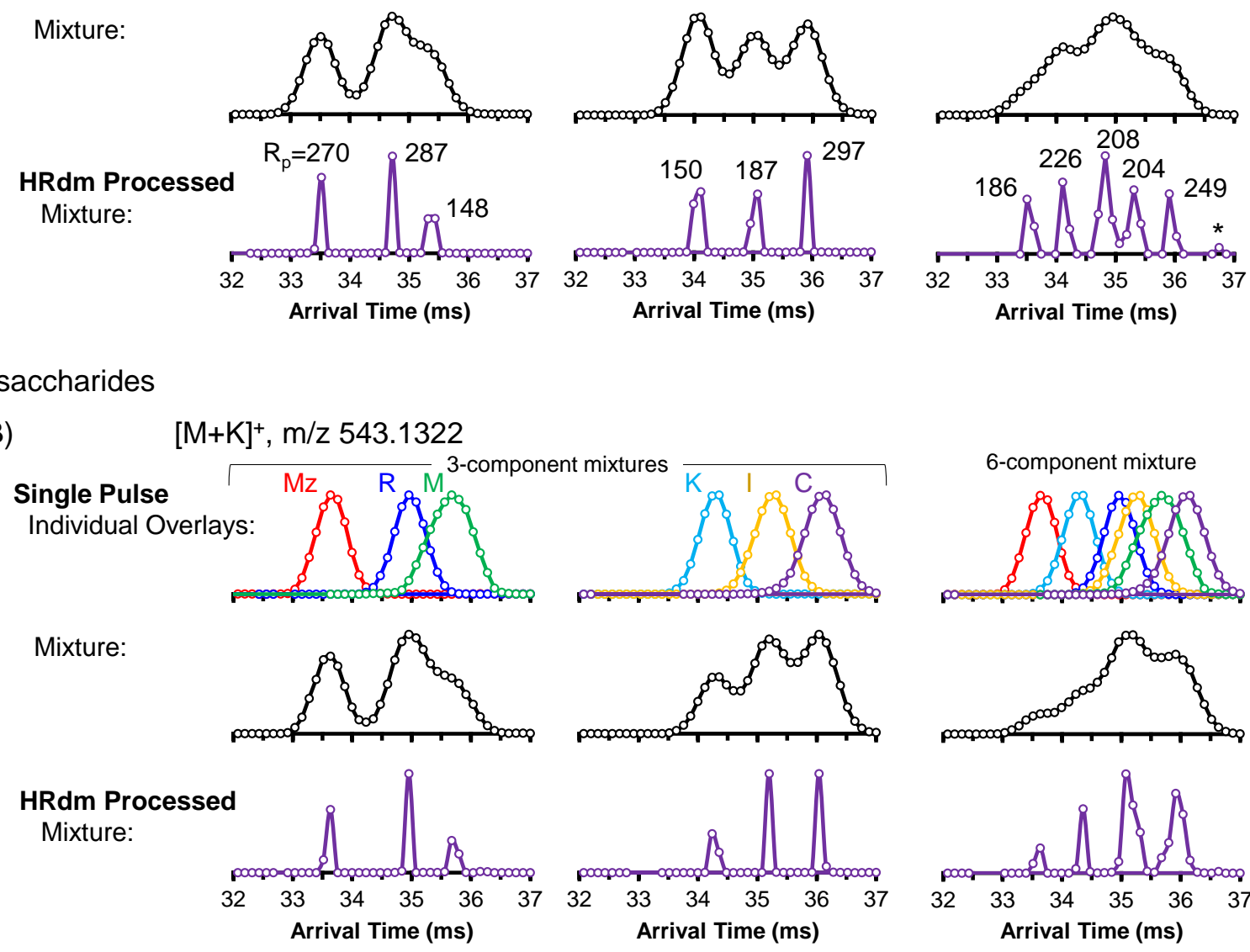

(C) IM Spectral Overlays for Mixtures - $[\mathrm{M}+\mathrm{Na}]^{+}, \mathrm{m} / \mathrm{z} 527.1582$
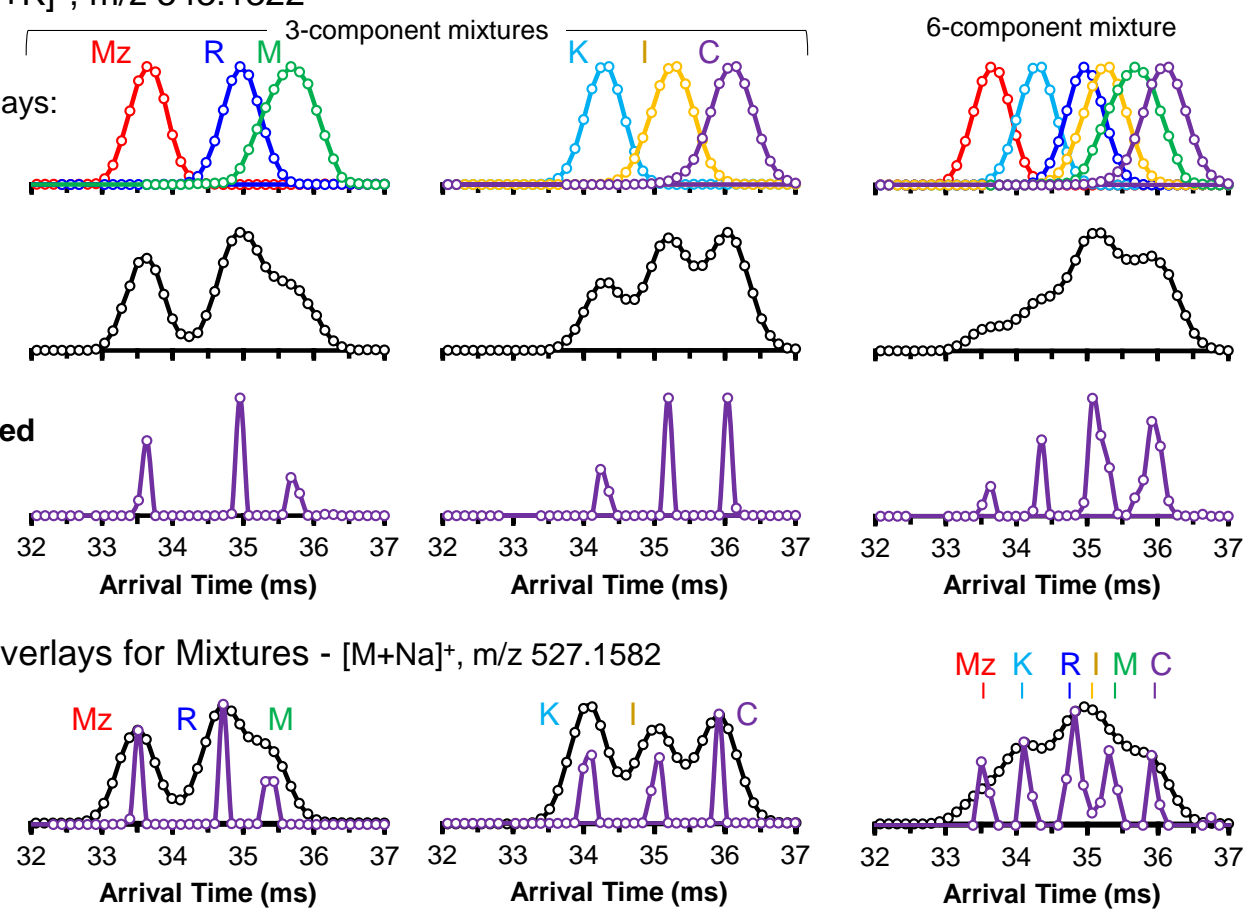

Figure S15. IM spectra (SP and HRdm) for the trisaccharide isomers corresponding to the (A) sodiumcoordinated ion forms, (B) potassium-coordinated ion forms, and (C) overlays of the $+\mathrm{Na}$ ions illustrating drift time alignment between single pulse and $\mathrm{HRdm}$ results of various 3 - and 6-component mixtures. 
Table S9. Summarized results for drift times, CCS measurements, \% differences in drift times $(\Delta P), R_{p}$, $\mathrm{R}_{\mathrm{pp}}$, and \% valley separation for pairwise comparisons of all isomers investigated in this study. For the trisaccharides, only results for the "nearest neighbor" isomer pairs are shown.

\begin{tabular}{|c|c|c|c|c|c|c|c|c|c|c|c|c|}
\hline \multirow{4}{*}{\multicolumn{2}{|c|}{$\begin{array}{c}\text { Isomer Pair } \\
\text { (Isomer } 1 \text { / Isomer 2) }\end{array}$}} & \multirow[b]{3}{*}{ Ion Form } & \multirow{3}{*}{ Z } & \multirow{3}{*}{$\begin{array}{l}\text { Exact lon } \\
\mathrm{m} / \mathrm{z}\end{array}$} & \multirow{2}{*}{\multicolumn{2}{|c|}{$\begin{array}{l}\text { Drift Time }\left(t_{d}\right)^{a} \\
{[m s]}\end{array}$}} & \multirow{2}{*}{\multicolumn{2}{|c|}{$\begin{array}{c}\text { CCS Valueb } \\
{\left[\AA^{2}\right]}\end{array}$}} & \multirow{3}{*}{$\begin{array}{l}\text { Percent } \\
\text { Difference } \\
\text { Eqn S5 }\end{array}$} & \multicolumn{2}{|c|}{ HRdm (highest, LVL3) } & \multirow{3}{*}{$\begin{array}{c}\text { Figure } \\
\text { Reference }\end{array}$} \\
\hline & & & & & & & & & & \multirow{2}{*}{$\begin{array}{c}R_{p p}{ }^{c} \\
\text { Eqn S2 }\end{array}$} & \multirow{2}{*}{$\begin{array}{l}\text { \% Valley } \\
\text { Separation }^{\mathrm{d}} \\
\text { Eqn S3 }\end{array}$} & \\
\hline & & & & & Iso. 1 & Iso. 2 & Iso. 1 & Iso. 2 & & & & \\
\hline & & {$[\mathrm{M}+\mathrm{H}]^{+}$} & 1 & 491.1970 & 33.55 & 33.83 & 203.54 & 205.43 & $0.9 \%$ & 0.9 & $25 \%$ & not shown \\
\hline \multirow{7}{*}{$\begin{array}{l}\text { do } \\
\frac{d}{0} \\
\frac{0}{0} \\
0 \\
0 \\
\frac{d}{0} \\
\frac{0}{0} \\
\frac{0}{0} \\
\frac{0}{\pi} \\
0\end{array}$} & & {$[\mathrm{M}+\mathrm{Na}]^{+}$} & 1 & 513.1790 & 33.40 & 34.09 & 202.40 & 206.61 & $2.1 \%$ & 3.5 & 100\% (baseline) & not shown \\
\hline & & {$[\mathrm{M}+\mathrm{K}]^{+}$} & 1 & 529.1529 & 34.32 & 34.76 & 207.81 & 210.50 & $1.3 \%$ & 1.6 & 100\% (baseline) & not shown \\
\hline & GD1b / GD1a (34:1) & {$[\mathrm{M}-2 \mathrm{H}]^{-2}$} & 2 & 903.4631 & 36.78 & 37.24 & 441.46 & 445.99 & $1.2 \%$ & 1.9 & 100\% (baseline) & Figure S9 \\
\hline & GD1b / GD1a (36:1) & {$[\mathrm{M}+2 \mathrm{Na}]^{+2}$} & 2 & 941.4753 & 38.17 & 39.61 & 454.63 & 471.70 & $3.7 \%$ & 4.9 & $100 \%$ (baseline) & Figure 4, S10 \\
\hline & & {$[\mathrm{M}-2 \mathrm{H}]^{-2}$} & 2 & 917.4788 & 37.18 & 37.41 & 446.30 & 448.98 & $0.6 \%$ & 0.8 & none & Figure S10 \\
\hline & GD1b / GD1a (38:1) & {$[\mathrm{M}+2 \mathrm{Na}]^{+2}$} & 2 & 955.4909 & 38.48 & 39.94 & 458.96 & 476.43 & $3.7 \%$ & 5.4 & 100\% (baseline) & Figure S11 \\
\hline & & {$[\mathrm{M}-2 \mathrm{H}]^{-2}$} & 2 & 931.4944 & 37.49 & 37.65 & 450.38 & 452.40 & $0.5 \%$ & 0.7 & $10 \%$ & Figure S11 \\
\hline \multirow{3}{*}{$\sum_{\text {İ }}^{n}$} & LNFP2 / LNFP1 & {$[\mathrm{M}+\mathrm{Na}]^{+}$} & 1 & 876.2955 & 44.59 & 45.54 & 268.98 & 274.42 & $2.0 \%$ & 2.9 & $100 \%$ (baseline) & Figure 4 \\
\hline & & {$[\mathrm{M}+\mathrm{K}]^{+}$} & 1 & 892.2695 & 45.21 & 45.54 & 272.45 & 274.83 & $0.9 \%$ & 1.2 & $45 \%$ & Figure S8 \\
\hline & & {$[\mathrm{M}+\mathrm{HK}]^{+2}$} & 2 & 446.6384 & 25.39 & 25.65 & 308.89 & 311.79 & $0.9 \%$ & 1.1 & $20 \%$ & not shown \\
\hline \multirow{6}{*}{$\sum^{n}$} & 1-LG / 2-LG & {$[\mathrm{M}+\mathrm{H}]^{+}$} & 1 & 355.2843 & 32.12 & 31.36 & 196.39 & 191.79 & $2.4 \%$ & 3.2 & 100\% (baseline) & not shown \\
\hline & & {$[\mathrm{M}+\mathrm{Na}]^{+}$} & 1 & 377.2662 & 31.57 & 31.79 & 192.27 & 193.59 & $0.8 \%$ & 1.1 & $75 \%$ & Figure 4 \\
\hline & & {$[\mathrm{M}+\mathrm{K}]^{+}$} & 1 & 393.2407 & 32.05 & 32.47 & 195.43 & 197.49 & $1.3 \%$ & 1.7 & 100\% (baseline) & not shown \\
\hline & $1-A G$ / 2-AG & {$[\mathrm{M}+\mathrm{H}]^{+}$} & 1 & 379.2842 & 32.45 & 32.54 & 197.54 & 198.04 & $0.3 \%$ & 0.4 & none & not shown \\
\hline & & {$[\mathrm{M}+\mathrm{Na}]^{+}$} & 1 & 401.2662 & 32.29 & 32.52 & 196.39 & 197.75 & $0.8 \%$ & 1.1 & $75 \%$ & Figure S12 \\
\hline & & {$[\mathrm{M}+\mathrm{K}]^{+}$} & 1 & 417.2402 & 32.81 & 33.13 & 199.18 & 201.09 & $1.1 \%$ & 1.4 & $95 \%$ & not shown \\
\hline \multirow{18}{*}{ 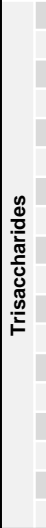 } & Melezitose / Kestose & {$[\mathrm{M}+\mathrm{Na}]^{+}$} & 1 & 527.1582 & 33.54 & 34.07 & 201.85 & 204.87 & $1.6 \%$ & 2.0 & $100 \%$ (baseline) & Figure 4, S13 \\
\hline & & {$[\mathrm{M}+\mathrm{K}]^{+}$} & 1 & 543.1322 & 33.65 & 34.29 & 202.33 & 206.04 & $1.9 \%$ & 2.5 & $100 \%$ (baseline) & Figure S13 \\
\hline & Melezitose / Raffinose & {$[\mathrm{M}+\mathrm{Na}]^{+}$} & 1 & 527.1582 & 33.54 & 34.78 & 201.85 & 208.82 & $3.5 \%$ & 4.9 & 100\% (baseline) & Figure 4, S13 \\
\hline & & {$[\mathrm{M}+\mathrm{K}]^{+}$} & 1 & 543.1322 & 33.65 & 34.97 & 202.33 & 209.96 & $3.8 \%$ & 6.4 & 100\% (baseline) & Figure S13 \\
\hline & Kestose / Raffinose & {$[\mathrm{M}+\mathrm{Na}]^{+}$} & 1 & 527.1582 & 34.07 & 34.78 & 204.87 & 208.82 & $2.0 \%$ & 2.6 & $100 \%$ (baseline) & Figure 4, S13 \\
\hline & & {$[\mathrm{M}+\mathrm{K}]^{+}$} & 1 & 543.1322 & 34.29 & 34.97 & 206.04 & 209.96 & $1.9 \%$ & 2.7 & 100\% (baseline) & Figure S13 \\
\hline & Kestose / Isomaltotriose & {$[\mathrm{M}+\mathrm{Na}]^{+}$} & 1 & 527.1582 & 34.07 & 35.06 & 204.87 & 210.83 & $2.9 \%$ & 3.9 & $100 \%$ (baseline) & Figure 4, S13 \\
\hline & & {$[\mathrm{M}+\mathrm{K}]^{+}$} & 1 & 543.1322 & 34.29 & 35.21 & 206.04 & 211.89 & $2.8 \%$ & 4.0 & 100\% (baseline) & Figure S13 \\
\hline & Raffinose / Isomaltotriose & {$[\mathrm{M}+\mathrm{Na}]^{+}$} & 1 & 527.1582 & 34.78 & 35.06 & 208.82 & 210.83 & $0.6 \%$ & 0.7 & unclear & Figure 4, S13 \\
\hline & & {$[\mathrm{M}+\mathrm{K}]^{+}$} & 1 & 543.1322 & 34.97 & 35.21 & 210.44 & 211.89 & $0.4 \%$ & 0.5 & unclear & Figure S13 \\
\hline & Raffinose / Maltotriose & {$[\mathrm{M}+\mathrm{Na}]^{+}$} & 1 & 527.1582 & 34.78 & 35.39 & 208.82 & 212.91 & $1.7 \%$ & 2.4 & $100 \%$ (baseline) & Figure 4, S13 \\
\hline & & {$[\mathrm{M}+\mathrm{K}]^{+}$} & 1 & 543.1322 & 34.97 & 35.67 & 209.96 & 214.46 & $2.0 \%$ & 3.1 & 100\% (baseline) & Figure S13 \\
\hline & Isomaltotriose / Maltotriose & {$[\mathrm{M}+\mathrm{Na}]^{+}$} & 1 & 527.1582 & 35.06 & 35.39 & 210.83 & 212.91 & $0.9 \%$ & 1.2 & unclear & Figure 4, S13 \\
\hline & & {$[\mathrm{M}+\mathrm{K}]^{+}$} & 1 & 543.1322 & 35.21 & 35.67 & 211.89 & 214.46 & $1.3 \%$ & 1.9 & unclear & Figure S13 \\
\hline & Isomaltotriose / Cellotriose & {$[\mathrm{M}+\mathrm{Na}]^{+}$} & 1 & 527.1582 & 35.06 & 35.88 & 210.83 & 216.07 & $2.3 \%$ & 3.6 & $100 \%$ (baseline) & Figure 4, S13 \\
\hline & & {$[\mathrm{M}+\mathrm{K}]^{+}$} & 1 & 543.1322 & 35.21 & 36.04 & 211.89 & 216.83 & $2.2 \%$ & 3.1 & 100\% (baseline) & Figure S13 \\
\hline & Maltotriose / Cellotriose & {$[\mathrm{M}+\mathrm{Na}]^{+}$} & 1 & 527.1582 & 35.39 & 35.88 & 212.91 & 216.07 & $1.4 \%$ & 1.8 & $100 \%$ (baseline) & Figure 4, S13 \\
\hline & & {$[\mathrm{M}+\mathrm{K}]^{+}$} & 1 & 543.1322 & 35.67 & 36.04 & 214.46 & 216.83 & $1.1 \%$ & 1.3 & $100 \%$ (baseline) & Figure S13 \\
\hline
\end{tabular}


(A)

(B)
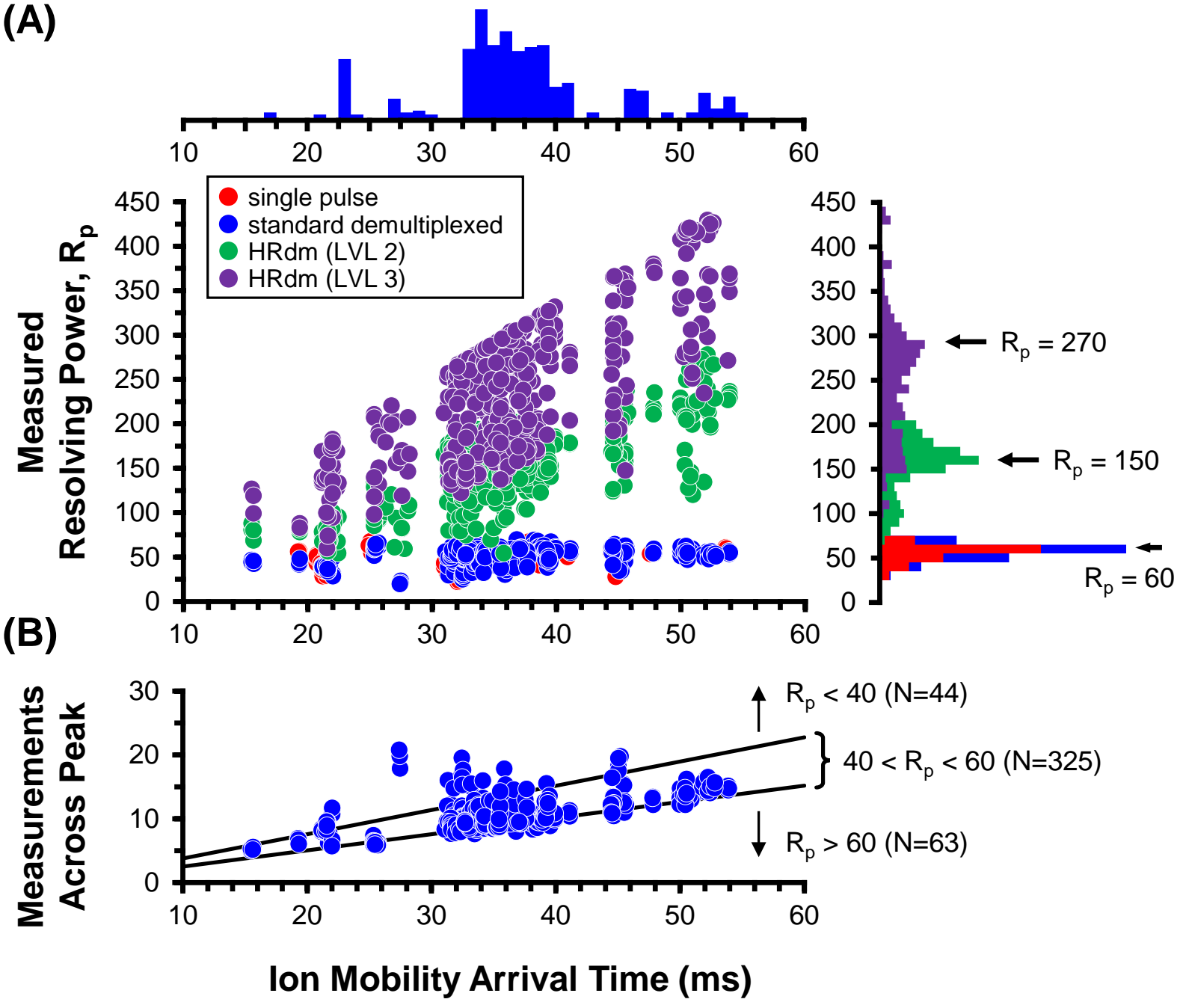

Figure S16. (A) A scatter plot of the measured resolving power $\left(R_{p}\right)$ as a function of $I M$ arrival time for single pulse, demultiplexed, and two processing levels of HRdm (level 2 "enhanced", and level 3 "highest"). The observed linear relationship of HRdm resolving power is a result of measuring peaks of constant width across increasingly higher drift times. The nonuniform distributions of data for HRdm are partly a result of fitting undersampled peaks, but also can be attributed to peaks which remain broad even after HRdm processing. This suggests these peaks possess unresolved fine structure, e.g., additional isomers/conformers. (B) The relationship between the drift time and the empirically determined number of data points across each drift peak for standard demultiplexed results. The data points falling higher than the theoretical resolving power boundaries are peaks exhibiting broader widths than would be expected by the resolving power accessed by the instrument. 


\section{4,039 Pairwise Comparisons of Empirical Entries in the CCS Compendium}

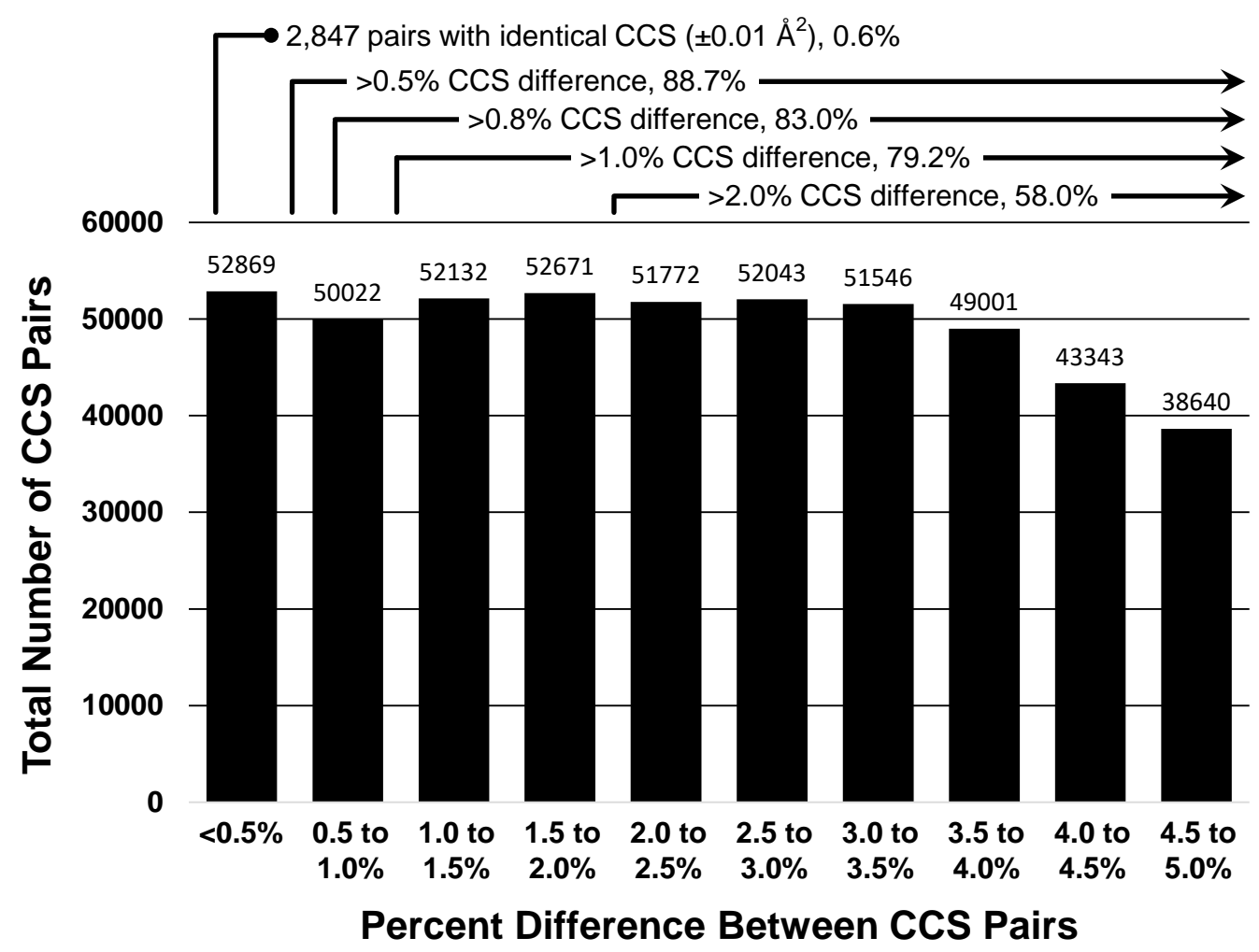

Figure S17. A "nearest neighbor" pairwise comparison of a large pool of empirical CCS measurements (CCS Compendium ${ }^{7}$ ) for CCS values falling with $\pm 5 \%$ of one another ( $N=494,039$ pairs). Analysis reveals that $83 \%$ of the pairs exhibit CCS differences larger than $0.8 \%$, which are potentially resolvable by HRdm. In contrast, a little over half (58\%) of the pairs exhibit a $>2 \%$ CCS difference that would potentially be resolvable using conventional DTIMS separation capabilities $\left(R_{p} \sim 60\right)$. 


\section{Appendix A - Ion Multiplexing and High Resolution Demultiplexing Protocol (concise).}

Materials - 6560 Ion Mobility-QTOF instrument (Agilent). Either MassHunter IM-MS Browser or MassHunter Mass Profiler software (Agilent). PreProcessor software (PNNL). High Resolution Demultiplexing software (Agilent).

Step 1 - Acquire multiplexed ion mobility data (4-bit and m/z 1700 mode recommended).

a) Tune and calibrate instrument in "IM-QTOF" mode as normal.

b) In the acquisition software, enable multiplexing by selecting "4-bit" in the drop-down menu (Q-TOF tab $\rightarrow$ Acquisition $\rightarrow$ Spectral Parameters $\rightarrow$ Multiplexing).

c) Next, set the "Trap Fill Time" (in the "IM Trap" section) to a value that is within the maximum allowed (maximum allowed is recommended) for the respective multiplex sequence.

Step 2-Demultiplex the data file using PNNL PreProcessor to reconstruct the IM spectrum.

a) In PreProcessor, load the data file to process and check "Demultiplexing". The default "Moving average window size" of 5 frames is recommended for most applications.

Step 3 - Ensure the demultiplexed files have the form "<name>.DeMP.d" (this is the default file name format from PreProcessor and is required for HRdm input).

Optional - Prior to Step 4, the user may wish to perform a mass recalibration (IM-MS Data File Reprocessing Utility) as well as a single-field CCS calibration (IM-MS Browser).

Step 4 - Generate a targeted Feature List using either IM-MS Browser or Mass Profiler.

For IM-MS Browser:

a) Load the demultiplexed data file into Browser and run the lon Mobility Feature Extractor by selecting "Find Features (IMFE)" under the "Method" menu.

b) Setup the method parameters in the IMFE popup window. This includes indicating the type of data file (Chromatographic or Infusion), the isotope model, the ion charge state limit, and the "Ion Intensity" threshold for feature finding. The "Report single-ion features with charge state $z=1$ " option is recommended, which assigns a charge state of 1 to isotopic envelopes that the software cannot confidently assign.

c) Select "Find Features" to generate the feature list.

d) Export the feature list as a .csv file by right clicking the "Feature List" and selecting "Export Table". Use "Data as a Text File", and "comma" delimiters. Ensure that this exported feature list is in the same file folder as the data file, which is necessary for HRdm processing (Step 5).

For Mass Profiler:

a) Create a new project (File $\rightarrow$ Create Project...), select "Number of groups", designate "Project name", and select "Input data type" as "*.d (MFE)", then "Add..." the DeMP files then "OK".

b) Select "Method Parameters" (refer to Step 4b), select "OK". Then "Run Current Method".

c) If prompted, select not to output average (consensus) values aligned across runs.

d) Export feature list (File $\rightarrow$ Export Composite-Compounds CEF) as a CEF file. Ensure that this exported feature list is in the same file folder as the data file.

Step 5 - Process the data file using HRdm.

a) In the High Resolution Demultiplexing software, load the demultiplexed data file (with the "DeMP" suffix). Three data files will appear for each file loaded.

b) Setup the options for HRdm. Under "Options..." choose the HR processing level, the MS width factor, and if a peak saturation check is performed (recommended).

c) Close the Options menu and select "Start HRdm" to initiate the file processing.

d) After processing, a new file will be written with the "HRdm" suffix. All information regarding the HRdm processing is saved to the file "HRdmLog.txt" located in the AcqData folder. The user may wish to designate in the file name which level of processing was performed. 


\section{References Cited for Supporting Information}

1. Dodds, J.N., May, J.C. and McLean, J.A., 2016. Investigation of the complete suite of the leucine and isoleucine isomers: toward prediction of ion mobility separation capabilities. Analytical Chemistry, 89(1), pp.952-959.

2. Dodds, J.N., May, J.C. and McLean, J.A., 2017. Correlating Resolving Power, Resolution, and Collision Cross Section: Unifying Cross-Platform Assessment of Separation Efficiency in Ion Mobility Spectrometry. Analytical Chemistry, 89(22), pp.12176-12184.

3. May, J.C., Dodds, J.N., Kurulugama, R.T., Stafford, G.C., Fjeldsted, J.C. and McLean, J.A., 2015. Broadscale resolving power performance of a high precision uniform field ion mobility-mass spectrometer. Analyst, 140(20), pp.6824-6833.

4. May, J.C., Goodwin, C.R., Lareau, N.M., Leaptrot, K.L., Morris, C.B., Kurulugama, R.T., Mordehai, A., Klein, C., Barry, W., Darland, E. and Overney, G., 2014. Conformational ordering of biomolecules in the gas phase: nitrogen collision cross sections measured on a prototype high resolution drift tube ion mobility-mass spectrometer. Analytical Chemistry, 86(4), pp.2107-2116.

5. Hines, K.M., May, J.C., McLean, J.A. and Xu, L., 2016. Evaluation of collision cross section calibrants for structural analysis of lipids by traveling wave ion mobility-mass spectrometry. Analytical Chemistry, 88(14), pp.7329-7336.

6. Stow, S.M., Causon, T.J., Zheng, X., Kurulugama, R.T., Mairinger, T., May, J.C., Rennie, E.E., Baker, E.S., Smith, R.D., McLean, J.A. and Hann, S., 2017. An interlaboratory evaluation of drift tube ion mobility-mass spectrometry collision cross section measurements. Analytical Chemistry, 89(17), pp.9048-9055.

7. Picache, J.A., Rose, B.S., Balinski, A., Leaptrot, K.L., Sherrod, S.D., May, J.C. and McLean, J.A., 2019. Collision cross section compendium to annotate and predict multi-omic compound identities. Chemical Science, 10(4), pp.983-993. Accessed November 04, 2019. 